\title{
Lectures on Spectrum Generating Symmetries and U-duality in Supergravity, Extremal Black Holes, Quantum Attractors and Harmonic Superspace
}

\author{
Murat Günaydin* \\ Institute for Gravitation and the Cosmos \\ Physics Department \\ Penn State University \\ University Park, PA 16802, USA
}

\begin{abstract}
We review the underlying algebraic structures of supergravity theories with symmetric scalar manifolds in five and four dimensions, orbits of their extremal black hole solutions and the spectrum generating extensions of their U-duality groups. For $5 D, N=2$ Maxwell-Einstein supergravity theories (MESGT) defined by Euclidean Jordan algebras,$J$, the spectrum generating symmetry groups are the conformal groups $C o n f(J)$ of $J$ which are isomorphic to their U-duality groups in four dimensions. Similarly, the spectrum generating symmetry groups of $4 D, N=2$ MESGTs are the quasiconformal groups $Q \operatorname{Conf}(J)$ associated with $J$ that are isomorphic to their U-duality groups in three dimensions. We then review the work on spectrum generating symmetries of spherically symmetric stationary $4 D$ BPS black holes, based on the equivalence of their attractor equations and the equations for geodesic motion of a fiducial particle on the target spaces of corresponding $3 D$ supergravity theories obtained by timelike reduction. We also discuss the connection between harmonic superspace formulation of $4 D, N=2$ sigma models coupled to supergravity and the minimal unitary representations of their isometry groups obtained by quantizing their quasiconformal realizations. We discuss the relevance of this connection to spectrum generating symmetries and conclude with a brief summary of more recent results.
\end{abstract}

KEYwords: Supergravity, Duality, Black Holes, Harmonic Superspace, Spectrum Generating Symmetries.

*murat@phys.psu.edu 


\section{Contents}

1. Introduction 2

2. U-duality Symmetries of Maximal Supergravity in various Dimensions 3

3. $5 D, N=2$ Maxwell-Einstein Supergravity Theories

田. MESGT's with Symmetric Target Spaces and Euclidean Jordan Algebras of Degree Three 6

5. Rotation ( Automorphism), Lorentz (Reduced Structure) and Conformal (Möbius) Groups of Jordan Algebras

6. U-duality Orbits of Extremal Black Hole Solutions of $5 D$ Supergravity Theories with Symmetric Target Manifolds and their Spectrum Generating Conformal Extensions

7. $4 \mathrm{D}, N=2$ Maxwell-Einstein Supergravity Theories with Symmetric Target Spaces and Freudenthal Triple Systems

8. U-duality Orbits of Extremal Black Holes of $4 D, N=2$ MESGTs with Symmetric Scalar Manifolds and of $N=8$ Supergravity and their Spectrum Generating Quasiconformal Extensions

9. Quasiconformal Realizations of Lie Groups and Freudenthal Triple Systems

10. $3 D$ U-duality Groups as Spectrum Generating Quasiconformal Groups of

$4 D$ Supergravity Theories and Quantum Attractor Flows

11. Harmonic Superspace, Minimal Unitary Representations and Quasiconformal Group Actions

$11.14 D, N=2 \sigma$-models coupled to Supergravity in Harmonic Superspace

11.2 Minimal Unitary Representations of Non-compact Groups from their Quasiconformal Realizations

11.3 Harmonic Superspace Formulation of $N=2$ Sigma Models and Minimal Unitary Representations of Their Isometry Groups

11.4 Implications

12. M/Superstring Theoretic Origins of $N=2$ MESGTs with Symmetric Scalar Manifolds 


\section{Introduction}

This review on spectrum generating symmetries in supergravity, extremal black holes, Uduality orbits, quantum attractor flows and harmonic superspace is based on four lectures given at the School on Attractor Mechanism (SAM 2007) in Frascati, Italy. The lectures were titled

1. Very special real geometry, Jordan algebras and attractors

2. Very special complex geometry, Freudenthal triple systems and attractors

3. Conformal and quasiconformal extensions of $U$-duality groups as spectrum generating symmetry groups

4. Harmonic superspace, quasiconformal groups and their minimal unitary representations. I will follow closely the material covered in the lectures and discuss briefly the results obtained since SAM 2007 at the end.

More specifically, section 2 is a review of the U-duality symmetries of maximal supergravity in various dimensions. In section 3 and 4 we review the $N=2$ Maxwell-Einstein supergravity theories (MESGT) in five dimensions and the connection between Jordan algebras of degree three and the MESGTs with symmetric scalar manifolds. This is followed by a review of the symmetry groups of Jordan algebras using the language of space-time symmetry groups and a complete list of simple finite dimensional Jordan algebras and their automorphism ( rotation), reduced structure ( Lorentz) and Möbius ( conformal) groups. In section 6 we review the U-duality orbits of extremal black holes of $5 D$ supergravity theories with symmetric target manifolds and discuss how these results lead to the proposal that $4 D$ U-duality groups act as spectrum generating symmetry groups of the corresponding $5 D$ theories. Section 7 is an overview of the $4 D$ MESGTs with symmetric target spaces and their connection with Freudenthal triple systems. This is followed by a classification of the U-duality orbits of extremal black holes of $N=2$ MESGTs with symmetric target manifolds and of $N=8$ supergravity and the proposal that the three dimensional U-duality groups act as spectrum generating quasiconformal groups of the corresponding $4 D$ theories. In section 9 we summarize the novel quasiconformal realizations of non-compact groups and their relation to Freudenthal triple systems. A precise and concrete implementation of the proposal that $3 D$ U-duality groups act as spectrum generating symmetry groups of the corresponding $4 D$ theories within the framework of spherically symmetric stationary BPS black holes is discussed in section 10. We then review the connection between the harmonic superspace formulation of $4 D, N=2$ sigma models coupled to supergravity and the minimal unitary representations of their isometry groups. For sigma models with symmetric target spaces we show that there is a remarkable map between the Killing potentials that generate their isom-

etry groups in harmonic superspace and the minimal unitary representations of these groups 
obtained by quantizing their quasiconformal realizations. Implications of this result are also discussed. Section12 is devoted to the M/superstring theoretic origins of $N=2$ MESGTs with symmetric target spaces, in particular, the magical supergravity theories. We conclude with a brief discussion of the related developments that took place since SAM 2007 and some open problems.

\section{U-duality Symmetries of Maximal Supergravity in various Dimensions}

The maximal possible dimension for Poincare supergravity is eleven dimensions [1]. Eleven dimensional supergravity involves a Majorana gravitino field, the elf-bein $E_{M}^{A}$ and an antisymmetric tensor of rank three $A_{M N P}$ and was constructed in [2]. Lagrangian of its bosonic sector has a very simple form

$$
\begin{aligned}
\mathcal{L}_{11}= & \frac{1}{\kappa_{11}^{2}}\left[-\frac{1}{2} E R-\frac{1}{48} E\left(F_{M N P Q}\right)^{2}\right. \\
& \left.-\frac{\sqrt{2}}{3456} \varepsilon^{M N P Q R S T U V W X} F_{M N P Q} F_{R S T U} A_{V W X}+\cdots\right]
\end{aligned}
$$

where the ellipses denote terms that involve fermions, $R$ is the scalar curvature, $E$ is the determinant of 11 dimensional elf-bein $E_{M}^{A}$ and $F_{M N P Q}$ is the field strength of the antisymmetric three-form field $A_{M N P}$ with $M, N, P, \ldots=0,1, \ldots ., 10$.

Under dimensional reduction to $d$ dimensions, eleven dimensional supergravity results in the maximal supergravity with a global symmetry group $E_{((11-d)(11-d))}$. We shall refer to these global symmetry groups as the U-duality groups even though the term was originally used for discrete subgroups of these continuous groups which are believed to be non-perturbative symmetries of $M$-theory toroidally compactified to $d$ dimensions [3].

In six dimensions maximal supergravity has the global symmetry group $E_{5(5)}=S O(5,5)$ and the scalar fields of this theory parametrize the symmetric space

$$
\mathcal{M}_{6}=\frac{S O(5,5)}{S O(5) \times S O(5)}
$$

In five dimensions the global symmetry group of maximal supergravity is $E_{6(6)}$ under which all the vector fields transform in the irreducible 27 dimensional representation of $E_{6(6)}$. The scalar fields of the theory parametrize the symmetric space

$$
\mathcal{M}_{5}=\frac{E_{6(6)}}{U S p(8)}
$$

U-duality group $E_{6(6)}$ is a symmetry of the Lagrangian of ungauged maximal supergravity in five dimensions.

In four dimensions the maximal ungauged supergravity has the U-duality group $E_{7(7)}$ as an on-shell symmetry under which field strengths of the 28 vector fields of the theory and their 
magnetic duals transform in the 56 dimensional representation, which is the smallest nontrivial representation of $E_{7(7)}$. The 70 scalar fields of the theory parametrize the symmetric space

$$
\mathcal{M}_{4}=\frac{E_{7(7)}}{S U(8)}
$$

Symmetry of the Lagrangian of ungauged $N=8$ supergravity theory in four dimensions depends on the real symplectic section chosen. Dimensional reduction from 5 dimensions leads to a symplectic section with $E_{6(6)} \times S O(1,1)$ symmetry of the Lagrangian such that under $E_{6(6)}$ electric field strengths transform in the reducible representation $(27+1)$. There exist also symplectic sections leading to Lagrangians with $S L(8, \mathbb{R})$ and $S U^{*}(8)$ symmetry groups. In the latter two cases the electric field strengths transform irreducibly in the 28 dimensional real representation of $S L(8, \mathbb{R})$ or $S U^{*}(8)$, respectively.

In three dimensions all the dynamical bosonic degrees of freedom of maximal supergravity can be dualized to scalar fields parametrizing the symmetric space [曰]

$$
\mathcal{M}_{3}=\frac{E_{8(8)}}{S O(16)}
$$

\section{3. $5 D, N=2$ Maxwell-Einstein Supergravity Theories}

Certain matter coupled supergravity theories do admit global symmetry groups which we shall also refer to as U-duality groups. In this section we shall study the U-duality groups that arise in five dimensional $N=2$ Maxwell-Einstein supergravity theories (MESGT). Five dimensional MESGTs that describe the coupling of an arbitrary number of $N=2$ (Abelian) vector multiplets to $N=2$ supergravity were constructed long ago in [5, 6, 7, 8]. The fields of the graviton supermultiplet are the fünfbein $e_{\mu}^{m}$, two gravitini $\psi_{\mu}^{i}(i=1,2)$, and a vector field $A_{\mu}$ ( the "bare" graviphoton). A vector multiplet consists of a vector field $A_{\mu}$, two "gaugini" $\lambda^{i}$ and one real scalar $\phi$. The bosonic part of five dimensional $N=2$ MESGT Lagrangian describing the coupling of $\left(n_{V}-1\right)$ vector multiplets has a very simple form ${ }^{1}$

$$
\begin{aligned}
e^{-1} \mathcal{L}_{\text {bosonic }}= & -\frac{1}{2} R-\frac{1}{4} \stackrel{\circ}{I J}_{I J} F_{\mu \nu}^{I} F^{J \mu \nu}-\frac{1}{2} g_{x y}\left(\partial_{\mu} \varphi^{x}\right)\left(\partial^{\mu} \varphi^{y}\right)+ \\
& +\frac{e^{-1}}{6 \sqrt{6}} C_{I J K} \varepsilon^{\mu \nu \rho \sigma \lambda} F_{\mu \nu}^{I} F_{\rho \sigma}^{J} A_{\lambda}^{K},
\end{aligned}
$$

where

$$
\begin{aligned}
I & =1, \ldots, n_{V} \\
x & =1, \ldots,\left(n_{V}-1\right) \\
\mu, \nu, \ldots & =0,1,2,3,4 .
\end{aligned}
$$

Note that we combined the "bare" graviphoton" with the other vector fields and labelled them with a single index $I$ which runs from 1 to $n_{V}$. $e$ and $R$ denote the fünfbein determinant

\footnotetext{
${ }^{1}$ We use the conventions of [6].
} 
and scalar curvature of spacetime, respectively. $F_{\mu \nu}^{I}$ are field strengths of the vector fields $A_{\mu}^{I}$. The metric, $g_{x y}$, of the scalar manifold $\mathcal{M}_{5}$ and the "metric" $\stackrel{\circ}{a}_{I J}$ of the kinetic energy term of vector fields both depend on the scalar fields $\varphi^{x}$. On the other hand, the completely symmetric tensor $C_{I J K}$ is constant as required by local Abelian gauge symmetries of vector fields.

Remarkably, the entire $5 D, N=2$ MESGT is uniquely determined by the constant tensor $C_{I J K}$ [6]. In particular, geometry of the scalar manifold $\mathcal{M}_{5}$ is determined by $C_{I J K}$ as follows. One defines a cubic polynomial, $\mathcal{V}(h)$, in $n_{V}$ real variables $h^{I}\left(I=1, \ldots, n_{V}\right)$ using the C-tensor,

$$
\mathcal{V}(h):=C_{I J K} h^{I} h^{J} h^{K} .
$$

and a metric, $a_{I J}$, of a $n_{V}$ dimensional ambient space $\mathcal{C}_{n_{V}}$ coordinatized by $h^{I}$ :

$$
a_{I J}(h):=-\frac{1}{3} \frac{\partial}{\partial h^{I}} \frac{\partial}{\partial h^{J}} \ln \mathcal{V}(h) .
$$

It can then be proven that the $\left(n_{V}-1\right)$-dimensional manifold, $\mathcal{M}_{5}$, of scalar fields $\varphi^{x}$ can be represented as an hypersurface defined by the condition [6]

$$
\mathcal{V}(h)=C_{I J K} h^{I} h^{J} h^{K}=1,
$$

in this ambient space $\mathcal{C}_{n_{V}}{ }^{2}$. The metric $g_{x y}$ is simply the pull-back of (3.3) to $\mathcal{M}_{5}$ :

$$
g_{x y}(\varphi)=\left.\left(h_{x}^{I} h_{y}^{J} a_{I J}\right)\right|_{\mathcal{V}=1}
$$

where

$$
h_{x}^{I}=\sqrt{\frac{3}{2}} \frac{\partial h^{I}}{\partial \phi^{x}} \mid \mathcal{V}=1
$$

and the "metric" $\stackrel{\circ}{a}_{I J}(\varphi)$ of kinetic energy term of the vector fields is given by the componentwise restriction of the metric $a_{I J}$ of the ambient space $\mathcal{C}_{n_{V}}$ to $\mathcal{M}_{5}$ :

$$
\stackrel{\circ}{a}_{I J}(\varphi)=a_{I J} \mid \mathcal{V}=1
$$

Riemann curvature tensor of the scalar manifold takes on a very simple form

$$
K_{x y z u}=\frac{4}{3}\left(g_{x[u} g_{z] y}+T_{x[u}{ }^{w} T_{z] y w}\right)
$$

where $T_{x y z}$ is a symmetric tensor that is the pull-back of the C-tensor to the hypersurface

$$
T_{x y z}=h_{x}^{I} h_{y}^{J} h_{z}^{K} C_{I J K}
$$

Since the Riemann curvature tensor $K_{x y z u}$ depends only on the metric $g_{x y}$ and the tensor $T_{x y z}$ it follows that the covariant constancy of $T_{x y z}$ implies the covariant constancy of $K_{x y z u}$ :

$$
T_{x y z ; w}=0 \longrightarrow K_{x y z u ; w}=0
$$

\footnotetext{
${ }^{2}$ The ambient space $\mathcal{C}_{n_{V}}$ is the 5 dimensional counterpart of the hyper Kähler cone of the twistor space of the corresponding three dimensional quaternionic geometry of the scalar manifold $\mathcal{M}_{3}$.
} 
Therefore scalar manifolds $\mathcal{M}_{5}$ with covariantly constant $T$ tensors are locally symmetric spaces. If the scalar manifold $\mathcal{M}_{5}$ is homogeneous then the covariant constancy of $T_{x y z}$ is equivalent to the "adjoint identity" for the C-tensor [6]:

$$
C^{I J K} C_{J(M N} C_{P Q) K}=\delta_{(M}^{I} C_{N P Q)}
$$

where the indices are raised by the inverse $\stackrel{\circ}{a}^{I J}$ of $\stackrel{\circ}{a}_{I J}$. Furthermore, cubic forms defined by $C_{I J K}$ of $N=2$ MESGT's that satisfy the adjoint identity and lead to positive definite metrics $g_{x y}$ and $\stackrel{\circ}{a}_{I J}(\varphi)$ are in one-to-one correspondence with norm forms of Euclidean (formally real) Jordan algebras $J$ of degree 3 [6]. The scalar manifolds of the corresponding MESGT's are of the form

$$
\mathcal{M}_{5}=\frac{\operatorname{Str}_{0}(J)}{\operatorname{Aut}(J)}
$$

where $\operatorname{Str}_{0}(J)$ is the invariance group of the norm $\mathbf{N}$ of $J$ and $A u t(J)$ is its automorphism group. These theories exhaust the list of $5 D$ MESGTs with symmetric target spaces $G / H$ such that $G$ is a symmetry of the Lagrangian [9]. Remarkably, the list of cubic forms that satisfy the adjoint identity coincides also with the list of Legendre invariant cubic forms that were classified more recently by mathematicians [10]. Before we discuss the geometries of $N=2$ MESGT's defined by Jordan algebras we shall take a detour and review some of the basic facts regarding Jordan algebras of degree three in the next subsection. For details and further references on Jordan algebras we refer to the monograph [1]

\section{MESGT's with Symmetric Target Spaces and Euclidean Jordan Algebras of Degree Three}

A Jordan algebra $J$ over a field $\mathbb{F}$ is a commutative and non-associative algebra with a product $\circ$ that satisfies

$$
X \circ Y=Y \circ X \in J, \quad \forall X, Y \in J
$$

and

$$
X \circ\left(Y \circ X^{2}\right)=(X \circ Y) \circ X^{2},
$$

where $X^{2} \equiv(X \circ X)$. Given a Jordan algebra $J$, one can define a norm form

$$
\mathbf{N}: J \rightarrow \mathbb{R}
$$

that satisfies the composition property [12]

$$
\mathbf{N}[2 X \circ(Y \circ X)-(X \circ X) \circ Y]=\mathbf{N}^{2}(X) \mathbf{N}(Y) .
$$

A Jordan algebra is said to be of degree, $p$, if its norm form satisfies $\mathbf{N}(\lambda X)=\lambda^{p} \mathbf{N}(X)$, where $\lambda \in \mathbb{R}$. A Euclidean Jordan algebra is a Jordan algebra for which the condition $X \circ X+Y \circ Y=0$ implies that $X=Y=0$ for all $X, Y \in J$. Euclidean Jordan algebras are sometimes called compact Jordan algebras since their automorphism groups are compact. 
As explained above, given a Euclidean Jordan algebra of degree three one can identify its norm form $\mathbf{N}$ with the cubic polynomial $\mathcal{V}$ defined by the C-tensor of a $5 \mathrm{D}, N=2$ MESGT with a symmetric scalar manifold [6]. Euclidean Jordan algebras of degree three fall into an infinite family of non-simple Jordan algebras which are direct sums of the form

$$
J=\mathbb{R} \oplus \Gamma_{(1, n-1)}
$$

where $\Gamma_{(1, n-1)}$ is an $n$ dimensional Jordan algebra of degree two associated with a quadratic norm form in $n$ dimensions that has a "Minkowskian signature" $(+,-, \ldots,-)$ and $\mathbb{R}$ is the one dimensional Jordan algebra. This infinite family of reducible Jordan algebras of degree 3 exists for any $n$ and is referred to as the generic Jordan family. The scalar manifolds of corresponding $5 \mathrm{D}, N=2$ MESGT's are

$$
\mathcal{M}_{5}\left(\mathbb{R} \oplus \Gamma_{(1, n-1)}\right)=\frac{S O(n-1,1)}{S O(n-1)} \times S O(1,1)
$$

An irreducible realization of $\Gamma_{(1, n-1)}$ is provided by $(n-1)\left(2^{[n / 2]} \times 2^{[n / 2]}\right)$ Dirac gamma matrices $\gamma^{i}(i, j, \ldots=1, \ldots,(n-1))$ of an $(n-1)$ dimensional Euclidean space together with the identity matrix $\gamma^{0}=\mathbf{1}$ and the Jordan product $\circ$ being defined as one half the anticommutator:

$$
\begin{aligned}
& \gamma^{i} \circ \gamma^{j}=\frac{1}{2}\left\{\gamma^{i}, \gamma^{j}\right\}=\delta^{i j} \gamma^{0} \\
& \gamma^{0} \circ \gamma^{0}=\frac{1}{2}\left\{\gamma^{0}, \gamma^{0}\right\}=\gamma^{0} \\
& \gamma^{i} \circ \gamma^{0}=\frac{1}{2}\left\{\gamma^{i}, \gamma^{0}\right\}=\gamma^{i} .
\end{aligned}
$$

The quadratic norm of a general element $\mathbb{X}=X_{0} \gamma^{0}+X_{i} \gamma^{i}$ of $\Gamma_{(1, n-1)}$ is defined as

$$
\mathbf{Q}(\mathbb{X})=\frac{1}{2^{[n / 2]}} \operatorname{Tr} \mathbb{X} \overline{\mathbb{X}}=X_{0} X_{0}-X_{i} X_{i},
$$

where

$$
\overline{\mathbb{X}} \equiv X_{0} \gamma^{0}-X_{i} \gamma^{i}
$$

The norm of a general element $y \oplus \mathbb{X}$ of the non-simple Jordan algebra $J=\mathbb{R} \oplus \Gamma_{(1, n-1)}$ is then simply

$$
\mathbf{N}(y \oplus \mathbb{X})=y \mathbf{Q}(\mathbb{X})
$$

where $y \in \mathbb{R}$.

In addition to this generic reducible infinite family, there exist four simple Euclidean Jordan algebras of degree three. They are realized by Hermitian $(3 \times 3)$-matrices over the four division algebras $\mathbb{A}=\mathbb{R}, \mathbb{C}, \mathbb{H}, \mathbb{O}$ ( reals $\mathbb{R}$, complex numbers $\mathbb{C}$, quaternions $\mathbb{H}$ and octonions $\mathbb{O}$.)

$$
J=\left(\begin{array}{ccc}
\alpha & Z & \bar{Y} \\
\bar{Z} & \beta & X \\
Y & \bar{X} & \gamma
\end{array}\right)
$$


where $\alpha, \beta, \gamma \in \mathbb{R}$ and $X, Y, Z \in \mathbb{A}$ with the Jordan product being one half the anticommutator. They are denoted as $J_{3}^{\mathbb{R}}, J_{3}^{\mathbb{C}}, J_{3}^{\mathbb{H}}, J_{3}^{\mathbb{Q}}$, respectively. The corresponding $N=2$ MESGTs are called "magical supergravity theories" [5]. The scalar manifolds of the magical supergravity theories in five dimensions are the irreducible symmetric spaces

$$
\begin{array}{ll}
J_{3}^{\mathbb{R}}: & \mathcal{M}=S L(3, \mathbb{R}) / S O(3) \\
J_{3}^{\mathbb{C}}: & \mathcal{M}=S L(3, \mathbb{C}) / S U(3) \\
J_{3}^{\mathbb{H}}: & \mathcal{M}=S U^{*}(6) / U S p(6) \\
J_{3}^{\mathbb{Q}}: & \mathcal{M}=E_{6(-26)} / F_{4}
\end{array}
$$

The cubic norm form, $\mathbf{N}$, of the simple Jordan algebras is given by the determinant of the corresponding Hermitian $(3 \times 3)$-matrices.

$$
\mathbf{N}(J)=\alpha \beta \gamma-\alpha X \bar{X}-\beta Y \bar{Y}-\gamma Z \bar{Z}+2 \operatorname{Re}(X Y Z)
$$

$R e(X Y Z)$ denotes the real part of $X Y Z$

$$
R e(X Y Z)=\operatorname{Re}(X(Y Z))=\operatorname{Re}((X Y) Z)=\frac{1}{2}(X Y Z+\overline{X Y Z})
$$

where bar denotes conjugation in the underlying division algebra.

A real quaternion $X \in \mathbb{H}$ can be expanded as

$$
\begin{aligned}
X & =X_{0}+X_{1} j_{1}+X_{2} j_{2}+X_{3} j_{3} \\
\bar{X} & =X_{0}-X_{1} j_{1}-X_{2} j_{2}-X_{3} j_{3} \\
X \bar{X} & =X_{0}^{2}+X_{1}^{2}+X_{2}^{2}+X_{3}^{2}
\end{aligned}
$$

where the imaginary units $j_{i}(i=1,2,3)$ satisfy

$$
j_{i} j_{j}=-\delta_{i j}+\epsilon_{i j k} j_{k}
$$

A real octonion $X \in \mathbb{O}$ has an expansion

$$
\begin{aligned}
X & =X_{0}+X_{1} j_{1}+X_{2} j_{2}+X_{3} j_{3}+X_{4} j_{4}+X_{5} j_{5}+X_{6} j_{6}+X_{7} j_{7} \\
\bar{X} & =X_{0}-X_{1} j_{1}-X_{2} j_{2}-X_{3} j_{3}-X_{4} j_{4}-X_{5} j_{5}-X_{6} j_{6}-X_{7} j_{7} \\
X \bar{X} & =X_{0}^{2}+\sum_{A=1}^{7}\left(X_{A}\right)^{2}
\end{aligned}
$$

where the seven imaginary units $j_{A}(A=1,2, \ldots, 7)$ satisfy

$$
j_{A} j_{B}=-\delta_{A B}+\eta_{A B C} j_{C}
$$

The $G_{2}$ invariant tensor $\eta_{A B C}$ is completely antisymmetric and in the conventions of [13] its nonvanishing components take on the values

$$
\eta_{A B C}=1 \Leftrightarrow(A B C)=(123),(471),(572),(673),(624),(435),(516)
$$


The bosonic content and scalar manifold of $N=6$ supergravity is the same as that of the $N=2$ MESGT defined by the simple Euclidean Jordan algebra $J_{3}^{\mathbb{H}}[5]$, namely

$$
\mathcal{M}_{5}=\frac{S U^{*}(6)}{U S p(6)}
$$

Therefore its invariant C-tensor is simply the one given by the cubic norm of $J_{3}^{\mathbb{H}}$.

The C-tensor $C_{I J K}$ of $N=8$ supergravity in five dimensions can be identified with the symmetric tensor given by the cubic norm of the split exceptional Jordan algebra $J_{3}^{\mathbb{O}_{s}} 14$, 15 defined over split octonions $\mathbb{O}_{s}$. The automorphism group of the split exceptional Jordan algebra defined by $3 \times 3$ Hermitian matrices over the split octonions $\mathbb{O}_{s}$

$$
J^{s}=\left(\begin{array}{ccc}
\alpha & Z^{s} & \bar{Y}^{s} \\
\bar{Z}^{s} & \beta & X^{s} \\
Y^{s} & \bar{X}^{s} & \gamma
\end{array}\right)
$$

,where $X^{s}, Y^{s}, Z^{S}$ are split octonions, is the noncompact group $F_{4(4)}$ with the maximal compact subgroup $U S p(6) \times U S p(2)$. Its reduced structure group is $E_{6(6)}$ under which the C-tensor is invariant. $E_{6(6)}$ is the U-duality group of maximal supergravity in five dimensions whose scalar manifold is

$$
E_{6(6)} / U S p(8)
$$

\section{Rotation (Automorphism), Lorentz (Reduced Structure) and Conformal (Möbius) Groups of Jordan Algebras}

Above we reviewed briefly the connections between Jordan algebras of degree three and supergravity theories. Jordan algebras were used in the very early days of spacetime supersymmetry to define generalized spacetimes that naturally extend the description of four dimensional Minkowski spacetime and its symmetry groups in terms of $2 \times 2$ complex Hermitian matrices. This was mainly motivated by attempts to find the super analogs of the exceptional Lie algebras [16] before a complete classification of finite dimensional simple Lie superalgebras was given by Kac 17.

As is well-known the twistor formalism in four-dimensional space-time $(d=4)$ leads naturally to the representation of spacetime coordinates $x_{\mu}$ in terms of $2 \times 2$ Hermitian matrices over the field of complex numbers $\mathbb{C}$ :

$$
x=x_{\mu} \sigma^{\mu}
$$

Hermitian matrices over the field of complex numbers close under the symmetric anti-commutator product and form a simple Jordan algebra denoted as $J_{2}^{\mathbb{C}}$. Therefore one can regard the four dimensional Minkowski coordinate vectors as elements of the Jordan algebra $J_{2}^{\mathbb{C}}$ 16, 18]. Then the rotation, Lorentz and conformal groups in four dimensions correspond simply to the automorphism, reduced structure and Möbius (linear fractional) groups of the Jordan algebra 


\begin{tabular}{|c|c|c|c|}
\hline$J$ & $\operatorname{Rot}(J)$ & $\operatorname{Lor}(J)$ & $\operatorname{Conf}(J)$ \\
\hline$J_{n}^{\mathbb{R}}$ & $S O(n)$ & $S L(n, \mathbb{R})$ & $S p(2 n, \mathbb{R})$ \\
$J_{n}^{\mathbb{C}}$ & $S U(n)$ & $S L(n, \mathbb{C})$ & $S U(n, n)$ \\
$J_{n}^{\mathbb{H}}$ & $U S p(2 n)$ & $S U^{*}(2 n)$ & $S O^{*}(4 n)$ \\
$J_{3}^{\mathbb{Q}}$ & $F_{4}$ & $E_{6(-26)}$ & $E_{7(-25)}$ \\
$\Gamma_{(1, d)}$ & $S O(d)$ & $S O(d, 1)$ & $S O(d, 2)$ \\
\hline
\end{tabular}

Table 1: Above we give the complete list of simple Euclidean Jordan algebras and their rotation ( automorphism), Lorentz ( reduced structure) and Conformal ( linear fractional) groups.

The symbols $\mathbb{R}, \mathbb{C}, \mathbb{H}, \mathbb{O}$ represent the four division algebras. For the Jordan algebras $J_{n}^{\mathbb{A}}$ of $n \times n$ hermitian matrices over $\mathbb{A}$ the norm form is the determinental form (or its generalization to the quaternionic and octonionic matrices).

$J_{2}^{\mathbb{C}}$ [16, 18]. The reduced structure group $\operatorname{Str}_{0}(J)$ of a Jordan algebra $J$ is simply the invariance group of its norm form $\mathbf{N}(J)$, while the structure group $\operatorname{Str}(J)=\operatorname{Str}_{0}(J) \times S O(1,1)$ is defined as the invariance group of $\mathbf{N}(J)$ modulo an overall nonzero global scale factor. This correspondence was then used to define generalized space-times coordinatized by elements of general Jordan algebras whose rotation $\operatorname{Rot}(J)$, Lorentz $\operatorname{Lor}(J)$ and conformal $\operatorname{Conf}(J)$

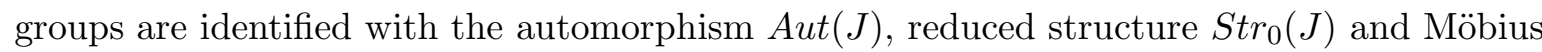
$\operatorname{Möb}(J)$ groups of $J$ [16, 18, 19, 20]. Denoting as $J_{n}^{\mathbb{A}}$ the Jordan algebra of $n \times n$ Hermitian matrices over the division algebra $\mathbb{A}$ and the Jordan algebra of Dirac gamma matrices in $d$ (Euclidean) dimensions as $\Gamma_{(1, d)}$ we list the symmetry groups of generalized space-times defined by simple Euclidean (formally real) Jordan algebras in Table 1

Note that for Euclidean Jordan algebras $\Gamma_{(1, d)}$ the automorphism, reduced structure and Möbius groups are simply the rotation, Lorentz and conformal groups of $(d+1)$-dimensional Minkowski spacetime.There exist the following special isomorphisms between the Jordan algebras of $2 \times 2$ Hermitian matrices over the four division algebras and the Jordan algebras of gamma matrices:

$$
J_{2}^{\mathbb{R}} \simeq \Gamma_{(1,2)} \quad ; \quad J_{2}^{\mathbb{C}} \simeq \Gamma_{(1,3)} \quad ; \quad J_{2}^{\mathbb{H}} \simeq \Gamma_{(1,5)} \quad ; \quad J_{2}^{\mathbb{Q}} \simeq \Gamma_{(1,9)}
$$

The Minkowski spacetimes they correspond to are precisely the critical dimensions for the existence of super Yang-Mills theories as well as of the classical Green-Schwarz superstrings. These Jordan algebras are all quadratic and their norm forms are precisely the quadratic invariants constructed using the Minkowski metric. The spacetimes defined by simple Jordan 
algebras of degree three can be interpreted as extensions of Minkowskian spacetimes in critical dimensions by bosonic spinorial coordinates plus a dilaton and the adjoint identity implies the Fierz identities for the existence of the corresponding supersymmetric theories [21, 22].

We should note two important facts about Table 1. First, the conformal groups of generalized space-times defined by Euclidean (formally real) Jordan algebras all admit positive energy unitary representations. Hence they can be given a causal structure with a unitary time evolution as in four dimensional Minkowski space-time [23, 24]. Second is the fact that the maximal compact subgroups of the generalized conformal groups of formally real Jordan algebras are simply the compact real forms of their structure groups (which are the products of their generalized Lorentz groups with dilatations).

Conformal group $\operatorname{Conf}(J)$ of a Jordan algebra $J$ is generated by translations $T_{\mathbf{a}}$, special conformal generators $K_{\mathbf{a}}$, dilatations and Lorentz transformations $M_{\mathbf{a b}}(\mathbf{a}, \mathbf{b} \in J)$. Lorentz transformations and dilatations generate the structure algebra $\mathfrak{s t r}(J)$ of $J$ [16, 19, 20]. Lie algebra $\operatorname{conf}(J)$ of the conformal group $C o n f(J)$ has a 3 -grading with respect to the generator $D$ of dilatations:

$$
\mathfrak{c o n f}(J)=K_{\mathbf{a}} \oplus M_{\mathbf{a b}} \oplus T_{\mathbf{b}}
$$

Action of $\operatorname{conf}(J)$ on the elements $\mathbf{x}$ of a Jordan algebra $J$ are as follows [20]:

$$
\begin{array}{r}
T_{\mathbf{a} \mathbf{x}}=\mathbf{a} \\
M_{\mathbf{a b}} \mathbf{x}=\{\mathbf{a b x}\} \\
K_{\mathbf{a}} \mathbf{x}=-\frac{1}{2}\{\mathbf{x a x}\}
\end{array}
$$

where $\{\mathbf{a b x}\}$ is the Jordan triple product

$$
\begin{gathered}
\{\mathbf{a b x}\}:=\mathbf{a} \circ(\mathbf{b} \circ \mathbf{x})-\mathbf{b} \circ(\mathbf{a} \cdot \mathbf{x})+(\mathbf{a} \circ \mathbf{b}) \circ \mathbf{x} \\
\mathbf{a}, \mathbf{b}, \mathbf{x} \in J
\end{gathered}
$$

with $\circ$ denoting the Jordan product. They satisfy the commutation relations

$$
\begin{aligned}
{\left[T_{\mathbf{a}}, K_{\mathbf{b}}\right] } & =M_{\mathbf{a b}} \\
{\left[M_{\mathbf{a b}}, T_{\mathbf{c}}\right] } & =T_{\{\mathbf{a b c}\}} \\
{\left[M_{\mathbf{a b}}, K_{\mathbf{c}}\right] } & =K_{\{\mathbf{b a c}\}} \\
{\left[M_{\mathbf{a b}}, M_{\mathbf{c d}}\right] } & =M_{\{\mathbf{a b c}\} \mathbf{d}}-M_{\{\mathbf{b a d}\} \mathbf{c}}
\end{aligned}
$$

corresponding to the well-known Tits-Kantor-Koecher construction of Lie algebras from Jordan triple systems [25, 26, 27]. The generators $M_{\mathbf{a b}}$ can be decomposed as

$$
M_{\mathbf{a b}}=D_{\mathbf{a}, \mathbf{b}}+L_{\mathbf{a} \cdot \mathbf{b}}
$$

where $D_{\mathbf{a}, \mathbf{b}}$ are the derivations that generate the automorphism (rotation) group of $J$

$$
D_{\mathbf{a}, \mathbf{b}} \mathbf{x}=\mathbf{a} \circ(\mathbf{b} \circ \mathbf{x})-\mathbf{b} \circ(\mathbf{a} \circ \mathbf{x})
$$


and $L_{c}$ denotes multiplication by the element $c \in J$. The dilatation generator $D$ is proportional to the multiplication operator by the identity element of $J$.

Choosing a basis $e_{I}$ and a conjugate basis $\tilde{e}^{I}$ of a Jordan algebra $J$ transforming covariantly and contravariantly, respectively, under the action of the Lorentz ( reduced structure) group of $J$ one can expand an element $\mathbf{x} \in J$ as

$$
\mathbf{x}=e_{I} q^{I}=\tilde{e}^{I} q_{I}
$$

In this basis one can write the generators of $\operatorname{con} \mathfrak{f}(J)$ as differential operators acting on the "coordinates" $q^{I}[20]$. These generators can be twisted by a unitary character $\lambda$ and take on a simple and elegant form

$$
\begin{aligned}
T_{I} & =\frac{\partial}{\partial q^{I}} \\
R_{J}^{I} & =-\Lambda_{J L}^{I K} q^{L} \frac{\partial}{\partial q^{K}}-\lambda \delta_{J}^{I} \\
K^{I} & =\frac{1}{2} \Lambda_{J L}^{I K} q^{J} q^{L} \frac{\partial}{\partial q^{K}}+\lambda q^{I}
\end{aligned}
$$

where

$$
\Lambda_{K L}^{I J}:=\delta_{K}^{I} \delta_{L}^{J}+\delta_{L}^{I} \delta_{K}^{J}-\frac{4}{3} C^{I J M} C_{K L M}
$$

They satisfy the commutation relations

$$
\begin{aligned}
{\left[T_{I}, K^{J}\right] } & =-R_{I}^{J} \\
{\left[R_{I}^{J}, T_{K}\right] } & =\Lambda_{I K}^{J L} T_{L} \\
{\left[R_{I}^{J}, K^{K}\right] } & =-\Lambda_{I K}^{J L} K^{L}
\end{aligned}
$$

The generator of the rotation (automorphism) subgroup are simply

$$
A_{I J}=R_{I}^{J}-R_{J}^{I}
$$

\section{U-duality Orbits of Extremal Black Hole Solutions of $5 D$ Supergravity Theories with Symmetric Target Manifolds and their Spectrum Gener- ating Conformal Extensions}

Orbits of the spherically symmetric stationary BPS black holes (BH) with non-vanishing entropy under the action of U-duality groups of $N=2$ MESGT's with symmetric target spaces were given in 14. In the same work the orbits with non-vanishing cubic invariants that are non-BPS were also classified. These latter orbits describe extremal non-BPS black holes and corresponding solutions to the attractor equations were given in 28]. In this section we shall review the solutions to the attractor equations in 5D MESGTs for extremal black holes, BPS as well as non-BPS, following [28]. 
Let us denote the $(n+1)$ dimensional charge vector in an extremal $\mathrm{BH}$ background as $q_{I}$. It is given by

$$
q_{I}=\int_{S^{3}} H_{I}=\int_{S^{3}} \stackrel{\circ}{a}_{I J} * F^{J} \quad(I=0,1, \ldots n)
$$

The black hole potential [29, 30] that determines the attractor flow takes on the following form for $N=2$ MESGTs:

$$
V(\phi, q)=q_{I}^{\stackrel{\circ}{I J}} q_{J}
$$

where $\stackrel{\circ}{a}^{I J}$ is the inverse of the metric $\stackrel{\circ}{a}_{I J}$ of the kinetic energy term of the vector fields. In terms of the central charge function

$$
Z=q_{I} h^{I}
$$

the potential can be written as

$$
V(q, \phi)=Z^{2}+\frac{3}{2} g^{x y} \partial_{x} Z \partial_{y} Z
$$

where

$$
\partial_{x} Z=q_{I} h_{, x}^{I}=\sqrt{\frac{2}{3}} h_{x}^{I}
$$

The critical points of the potential are determined by the equation

$$
\partial_{x} V=2\left(2 Z \partial_{x} Z-\sqrt{3 / 2} T_{x y z} g^{y y^{\prime}} g^{z z^{\prime}} \partial_{y^{\prime}} Z \partial_{z^{\prime}} Z\right)=0
$$

The BPS attractors are given by the solutions satisfying [31, 32]

$$
\partial_{x} Z=0
$$

at the critical points. The non-BPS attractors are given by non-trivial solutions 28]

$$
2 Z \partial_{x} Z=\sqrt{\frac{3}{2}} T_{x y z} \partial^{y} Z \partial^{z} Z
$$

such that

$$
\partial^{x} Z \equiv g^{x x^{\prime}} \partial_{x^{\prime}} Z \neq 0
$$

at the critical points. The equation 6.6 can be inverted using the relation

$$
q_{I}=h_{I} Z-\frac{3}{2} h_{I, x} \partial^{x} Z
$$

For BPS attractors satisfying $\partial_{x} Z=0$ this gives

$$
q_{I}=h_{I} Z
$$

and for non-BPS attractors satisfying $\partial_{x} Z \neq 0$ we get

$$
q_{I}=h_{I} Z-(3 / 2)^{3 / 2} \frac{1}{2 Z} h_{I, x} T^{x y z} \partial_{y} Z \partial_{z} Z
$$




\begin{tabular}{|c|c|}
\hline$J$ & $\mathcal{O}_{B P S}=\operatorname{Str}_{0}(J) / A u t(J)$ \\
\hline$J_{3}^{\mathbb{R}}$ & $S L(3, \mathbb{R}) / S O(3)$ \\
\hline$J_{3}^{\mathbb{C}}$ & $S L(3, \mathbb{C}) / S U(3)$ \\
\hline$J_{3}^{\mathbb{H}}$ & $S U^{*}(6) / U S p(6)$ \\
\hline$J_{3}^{\bigotimes}$ & $E_{6(-26)} / F_{4}$ \\
\hline $\mathbb{R} \oplus \Gamma_{(1, n-1)}$ & $S O(n-1,1) \times S O(1,1) / S O(n-1)$ \\
\hline
\end{tabular}

Table 2: Above we list the orbits of spherically symmetric stationary BPS black hole solutions in $5 D$ MESGTs defined by Euclidean Jordan algebras $J$ of degree three. U-duality and stability groups are given by the Lorentz ( reduced structure ) and rotation ( automorphism) groups of $J$.

\begin{tabular}{|c|c|c|}
\hline$J$ & $\mathcal{O}_{\text {non-BPS }}=\operatorname{Str}_{0}(J) / A u t\left(J_{(1,2)}\right)$ & $K \subset A u t\left(J_{(1,2)}\right)$ \\
\hline$J_{3}^{\mathbb{R}}$ & $S L(3, \mathbb{R}) / S O(2,1)$ & $\mathrm{SO}(2)$ \\
\hline$J_{3}^{\mathbb{C}}$ & $S L(3, \mathbb{C}) / S U(2,1)$ & $S U(2) \times U(1)$ \\
\hline$J_{3}^{\mathbb{H}}$ & $S U^{*}(6) / U S p(4,2)$ & $U S p(4) \times U S p(2)$ \\
\hline$J_{3}^{\bigotimes}$ & $E_{6(-26)} / F_{4(-20)}$ & $S O(9)$ \\
\hline $\mathbb{R} \oplus \Gamma_{(1, n-1)}$ & $S O(n-1,1) \times S O(1,1) / S O(n-2,1)$ & $S O(n-2)$ \\
\hline
\end{tabular}

Table 3: Above we list the orbits of non-BPS extremal black holes of $N=2$ MESGT's with nonvanishing entropy in $d=5$. The first column lists the Jordan algebras of degree 3 that define these theories. The third column lists the maximal compact subgroups $K$ of the stability group $\operatorname{Aut}\left(J_{(1,2)}\right)$.

Since the BPS attractor solution with non-vanishing entropy [31, 32] is given by $\partial_{x} Z=0$ , which is invariant under the automorphism group $A u t(J)$ of the underlying Jordan algebra $J$, the orbits of BPS black hole solutions are of the form

$$
\mathcal{O}_{B P S}=\operatorname{Str}_{0}(J) / A u t(J)
$$

and were listed in column 1 of table 1 of 14] which we reproduce in Table 2

The orbits for extremal non-BPS black holes with non-vanishing entropy are of the form

$$
\mathcal{O}_{\text {non-BPS }}=\operatorname{Str}_{0}(J) / \operatorname{Aut}\left(J_{(1,2)}\right)
$$

where $\operatorname{Aut}\left(J_{(1,2)}\right)$ is a noncompact real form of the automorphism group of $J$ and were listed in column 2 of table 1 of 14, which we reproduce in Table 3 .

The entropy $S$ of an extremal black hole solution of $N=2$ MESGT with charges $q_{I}$ is determined by the value of the black hole potential $V$ at the attractor points

$$
S=\left(V_{\text {critical }}\right)^{3 / 4}
$$

For $N=2$ MESGTs defined by Jordan algebras of degree 3 , the tensor $C_{I J K}$ is an invariant tensor of the U-duality group $\operatorname{Str}_{0}(J)$. Similarly the tensor $T_{a b c}$ with " flat" indices

$$
T_{a b c}=e_{a}^{x} e_{b}^{y} e_{c}^{z} T_{x y z}
$$


where $e_{a}^{x}$ is the $n$-bein on the $n$-dimensional scalar manifold with metric $g_{x y}$ is an invariant tensor of the maximal compact subgroup $\operatorname{Aut}(J)$ of $\operatorname{Str}_{0}(J)$. In terms of flat indices the attractor equation becomes:

$$
2 Z \partial_{a} Z=\sqrt{3 / 2} T_{a b c} \partial^{b} Z \partial^{c} Z
$$

Thus for BPS attractor solution $\partial_{a} Z=0$ one finds

$$
S_{B P S}=\left(V_{B P S}\right)^{3 / 4}=Z_{B P S}^{3 / 2}
$$

For extremal non-BPS attractors $\partial_{a} Z \neq 0$, squaring the criticality condition one finds

$$
4 Z^{2} \partial_{a} Z \partial_{a} Z=\frac{3}{2} T_{a b c} T_{a b^{\prime} c^{\prime}} \partial_{b} Z \partial_{c} Z \partial_{b}^{\prime} Z \partial_{c}^{\prime} Z
$$

Then using the identity

$$
T_{a(b c} T_{\left.b^{\prime} c^{\prime}\right)}^{a}=\frac{1}{2} g_{(b c} g_{\left.b^{\prime} c^{\prime}\right)}
$$

valid only for MESGTs defined by Jordan algebras of degree three one obtains

$$
\partial_{a} Z \partial_{a} Z=\frac{16}{3} Z^{2}
$$

Hence the entropy of extremal non-BPS black holes are given by ${ }^{3}$

$$
S_{n o n-B P S}=V_{n o n-B P S}^{4 / 3}=\left(Z^{2}+\frac{3}{2} \partial_{a} Z \partial_{a} Z\right)^{3 / 4}=\left(3 Z_{n o n-B P S}\right)^{3 / 2}
$$

By differentiating equation 6.4 one finds a general expression for the Hessian of the black hole potential around the critical points

$$
\begin{aligned}
\frac{1}{4} D_{x} \partial_{y} V= & \frac{2}{3} g_{x y} Z^{2}+\partial_{x} Z \partial_{y} Z-2 \sqrt{\frac{2}{3}} T_{x y z} g^{z w} \partial_{w} Z Z \\
& +T_{x p q} T_{y z s} g^{p z} g^{q q^{\prime}} g^{s s^{\prime}} \partial_{q^{\prime}} Z \partial_{s^{\prime}} Z \\
= & \frac{2}{3}\left(g_{x z} Z-\sqrt{\frac{3}{2}} T_{x z p} \partial^{p} Z\right)\left(g_{y z} Z-\sqrt{\frac{3}{2}} T_{y z q} \partial^{q} Z\right)+\partial_{x} Z \partial_{y} Z
\end{aligned}
$$

Thus for BPS critical points for which we have $\partial_{x} Z=0$ the Hessian is given simply as

$$
\partial_{x} \partial_{y} V=\frac{8}{3} g_{x y} Z^{2}
$$

which is the same result as in $d=4$ [30]. Since the metric of the scalar manifold is positive definite the above formula implies that the scalar fluctuations have positive square mass reflecting the the attractor nature of the BPS critical points.

\footnotetext{
${ }^{3}$ We should stress that both for BPS as well as extremal non-BPS black holes the quantities appearing in the above formulas are evaluated at the corresponding attractor points.
} 
For non-BPS extremal critical points of the black hole potential the Hessian has flat directions and is positive semi-definite [28].

The orbits of BPS black hole solutions of $N=8$ supergravity theory in five dimensions were also given in [14]. The 1/8 BPS black holes with non-vanishing entropy has the orbit

$$
\mathcal{O}_{1 / 8-B P S}=\frac{E_{6(6)}}{F_{4(4)}}=\frac{\operatorname{Str}_{0}\left(J_{3}^{\mathbb{Q}_{S}}\right)}{\operatorname{Aut}\left(J_{3}^{\mathbb{Q}_{S}}\right)}
$$

where $\mathbb{O}_{S}$ stands for the split octonions and $J_{3}^{\mathbb{Q}_{S}}$ is the split exceptional Jordan algebra. Note that in contrast to the exceptional $N=2$ MESGT theory defined by the real exceptional Jordan algebra that has two different orbits with nonvanishing entropy the maximal supergravity has only one such orbit. On the other hand maximal supergravity theory admits $1 / 4$ and $1 / 2$ BPS black hole solutions with vanishing entropy [33]. Their orbits under U-duality are 14]

$$
\begin{aligned}
\mathcal{O}_{1 / 4-B P S} & =\frac{E_{6(6)}}{O(5,4)\left(S T_{16}\right.} \\
\mathcal{O}_{1 / 2-B P S} & =\frac{E_{6(6)}}{O(5,5)\left(S T_{16}\right.}
\end{aligned}
$$

where (S) denotes the semi-direct product and $T_{16}$ is the group of translations transforming in the spinor $(16)$ of $S O(5,5)$. Vanishing entropy means vanishing cubic norm. Thus the black hole solutions corresponding to vanishing entropy has additional symmetries beyond the five dimensional U-duality group, namely they are invariant under the generalized special conformal transformations of the underlying Jordan algebras. This is complete parallel to the invariance of light-like vectors under special conformal transformations in four dimensional Minkowski spacetime which can be coordinatized by the elements of the Jordan algebra $J_{2}^{\mathbb{C}}$. Acting on a black hole solution with non-vanishing entropy these special conformal transformations change their norms and hence the entropy. Hence the conformal groups of Jordan algebras were proposed as spectrum generating symmetry groups of black hole solutions of MESGTs defined by them [14, 15, 34, 35. Since the conformal groups of Jordan algebras of degree three are isomorphic to the U-duality groups of the corresponding four dimensional supergravity theories obtained by dimensional reduction this implies that the four dimensional U-duality groups must act as spectrum generating symmetry groups of the corresponding five dimensional theories. Since it was first made, there have been several works relating black hole solutions in four and five dimensions ( $4 D / 5 D$ lift) [36, 37, 38, 39] that lend support to the proposal that four dimensional U-duality groups act as spectrum generating conformal symmetry groups of five dimensional supergravity theories from which they descend.

\section{4D, $N=2$ Maxwell-Einstein Supergravity Theories with Symmetric Tar- get Spaces and Freudenthal Triple Systems}

Under dimensional reduction on a torus $5 D, N=2$ MESGTs with $\left(n_{V}-1\right)$ vector multiplets 
lead to $4 D, N=2$ MESGTs with $n_{V}$ vector multiplets, with the extra vector multiplet coming from the $5 D$ graviton supermultiplet. The metric of the target space of the four-dimensional scalar fields of dimensionally reduced theories were first given in [6] in the so-called unbounded realization of their geometries. More precisely, the resulting four dimensional target spaces are generalized upper half-spaces (tube domains) over the convex cones defined by the cubic norm. They are parameterized by complex coordinates [6],

$$
z^{I}:=\frac{1}{\sqrt{3}} A^{I}+\frac{i}{\sqrt{2}} \tilde{h}^{I}
$$

where $A^{I}$ denote the $4 D$ scalars descending from the $5 D$ vectors. Imaginary components of $z^{I}$ are given by

$$
\tilde{h}^{I}:=e^{\sigma} h^{I}
$$

where $\sigma$ is the scalar field ( dilaton ) coming from $5 D$ graviton and $h^{I}$ were defined above. They satisfy the positivity condition

$$
\mathcal{V}\left(\tilde{h}^{I}\right)=C_{I J K} \tilde{h}^{I} \tilde{h}^{J} \tilde{h}^{K}=e^{3 \sigma}>0
$$

Geometry of four dimensional $N=2$ MESGTs obtained by dimensional reduction from five dimensions (R-map) was later referred to as "very special geometry" and studied extensively ${ }^{4}$. The full bosonic sector of $4 D$ theories obtained by dimensional reduction from gauged $5 D, N=2$ Yang-Mills Einstein supergravity coupled to tensor multiplets and their reformulation in the standard language of special Kähler geometry was given in [41], which we follow in our summary here, restricting ourselves to the ungauged MESGT theory without tensors.

As is well-known one can interpret the $n_{V}$ complex coordinates $z^{I}$ of dimensionally reduced MESGTs as inhomogeneous coordinates of a $\left(n_{V}+1\right)$-dimensional complex vector with coordinates $X^{A}$

$$
X^{A}=\left(\begin{array}{c}
X^{0} \\
X^{I}
\end{array}\right)=\left(\begin{array}{c}
1 \\
z^{I}
\end{array}\right) .
$$

where the capital Latin indices $A, B, C, \cdots$ run from 0 to $n_{V}$. Taking as "prepotential" the cubic form defined by the C-tensor coming from 5 dimensions

$$
F\left(X^{A}\right)=-\frac{\sqrt{2}}{3} C_{I J K} \frac{X^{I} X^{J} X^{K}}{X^{0}}
$$

and using the symplectic section

$$
\left(\begin{array}{c}
X^{A} \\
F_{A}
\end{array}\right)=\left(\begin{array}{c}
X^{A} \\
\partial_{A} F
\end{array}\right) \equiv\left(\begin{array}{c}
X^{A} \\
\frac{\partial F}{\partial X^{A}}
\end{array}\right)
$$

\footnotetext{
${ }^{4}$ See for example [4] and the references therein.
} 
one gets the Kähler potential

$$
\begin{aligned}
\mathcal{K}(X, \bar{X}) & :=-\ln \left[i \bar{X}^{A} F_{A}-i X^{A} \bar{F}_{A}\right] \\
& =-\ln \left[i \frac{\sqrt{2}}{3} C_{I J K}\left(z^{I}-\bar{z}^{I}\right)\left(z^{J}-\bar{z}^{J}\right)\left(z^{K}-\bar{z}^{K}\right)\right]
\end{aligned}
$$

which agrees precisely with the Kähler potential obtained in [6]. The "period matrix" that determines the kinetic terms of the vector fields in four dimensions is given by

$$
\mathcal{N}_{A B}:=\bar{F}_{A B}+2 i \frac{\operatorname{Im}\left(F_{A C}\right) \operatorname{Im}\left(F_{B D}\right) X^{C} X^{D}}{\operatorname{Im}\left(F_{C D}\right) X^{C} X^{D}}
$$

where $F_{A B} \equiv \partial_{A} \partial_{B} F$ etc. Components of the resulting period matrix $\mathcal{N}_{A B}$ under dimensional reduction are:

$$
\begin{aligned}
& \mathcal{N}_{00}=-\frac{2 \sqrt{2}}{9 \sqrt{3}} C_{I J K} A^{I} A^{J} A^{K}-\frac{i}{3}\left(e^{\sigma} \stackrel{\circ}{I J}_{I J} A^{I} A^{J}+\frac{1}{2} e^{3 \sigma}\right) \\
& \mathcal{N}_{0 I}=\frac{\sqrt{2}}{3} C_{I J K} A^{J} A^{K}+\frac{i}{\sqrt{3}} e^{\sigma^{\circ}} a_{I J} A^{J} \\
& \mathcal{N}_{I J}=-\frac{2 \sqrt{2}}{\sqrt{3}} C_{I J K} A^{K}-i e^{\sigma} \stackrel{\circ}{I J}_{I J}
\end{aligned}
$$

The prepotential (7.4) leads to the Kähler metric

$$
g_{I \bar{J}} \equiv \partial_{I} \partial_{\bar{J}} \mathcal{K}=\frac{3}{2} e^{-2 \sigma^{\circ}}{ }_{I J}
$$

for the scalar manifold $\mathcal{M}_{4}$ of four-dimensional theory, where $\stackrel{\circ}{a}_{I J}$ is the "metric" of the kinetic energy term of the vector fields of the $5 D$ theory. Above we denoted the field strength of the vector field that comes from the graviton in five dimensions as $F_{\mu \nu}^{0}$. The bosonic sector of dimensionally reduced Lagrangian can then be written as

$$
\begin{aligned}
e^{-1} \mathcal{L}^{(4)}= & -\frac{1}{2} R-g_{I \bar{J}}\left(\partial_{\mu} z^{I}\right)\left(\partial^{\mu} \bar{z}^{J}\right) \\
& +\frac{1}{4} \operatorname{Im}\left(\mathcal{N}_{A B}\right) F_{\mu \nu}^{A} F^{\mu \nu B}-\frac{1}{8} \operatorname{Re}\left(\mathcal{N}_{A B}\right) \epsilon^{\mu \nu \rho \sigma} F_{\mu \nu}^{A} F_{\rho \sigma}^{B} .
\end{aligned}
$$

Since the complex scalar fields $z^{I}$ of the four dimensional theory are restricted to the domain $\mathcal{V}(\operatorname{Im}(z))>0$, the scalar manifolds of $4 \mathrm{D}, N=2$ MESGT's defined by Euclidean Jordan algebras $J$ of degree three are simply the Köcher "upper half spaces" of the corresponding Jordan algebras, which belong to the family of Siegel domains of the first kind [42]. The "upper half spaces" of Jordan algebras can be mapped into bounded symmetric domains, which can be realized as hermitian symmetric spaces of the form

$$
\mathcal{M}_{4}=\frac{\operatorname{Conf}(J)}{\widetilde{S t r} J}
$$


where $\operatorname{Conf}(J)$ is the conformal group of the Jordan algebra $J$ and its maximal compact subgroup $\widetilde{S t r} J$ is the compact real form of its structure group $\operatorname{Str}(J)$. The Kähler potential 7.6 that one obtains directly under dimensional reduction from 5 dimensions is given by the "cubic light-cone"

$$
\mathcal{V}(z-\bar{z})=C_{I J K}\left(z^{I}-\bar{z}^{I}\right)\left(z^{J}-\bar{z}^{J}\right)\left(z^{K}-\bar{z}^{K}\right)
$$

which is manifestly invariant under the 5 dimensional U-duality group $\operatorname{Str}_{0}(J)$ and real translations

$$
\begin{gathered}
\operatorname{Re}\left(z^{I}\right) \Rightarrow \operatorname{Re}\left(z^{I}\right)+a^{I} \\
a^{I} \in \mathbb{R}
\end{gathered}
$$

which follows from Abelian gauge invariances of vector fields of the five dimensional theory. Under dilatations it gets simply rescaled. Infinitesimal action of special conformal generators $K^{I}$ of $C o n f(J)$ on the "cubic light-cone" yields [15]

$$
K^{I} \mathcal{V}(z-\bar{z})=\left(z^{I}+\bar{z}^{I}\right) \mathcal{V}(z-\bar{z})
$$

which can be integrated to give the global transformation of the form

$$
\mathcal{V}(z-\bar{z}) \Longrightarrow f\left(z^{I}\right) \bar{f}\left(\bar{z}^{I}\right) \mathcal{V}(z-\bar{z})
$$

This shows that the cubic light-cone defined by $\mathcal{V}(z-\bar{z})=0$ is invariant under the full conformal group $\operatorname{Con} f(J)$. Furthermore, the above global conformal group action leaves the metric $g_{I \bar{J}}$ invariant since it simply induces a Kähler transformation of the Kähler potential $\ln \mathcal{V}(z-\bar{z})$.

In $N=2$ MESGTs defined by Euclidean Jordan algebras $J$ of degree three, one-to-one correspondence between vector fields of five dimensional theories (and hence their charges) and elements of $J$ gets extended, in four dimensions, to a one-to-one correspondence between field strengths of vector fields plus their magnetic duals and Freudenthal triple systems defined over $J$ [6, 15, 14, 34, 22]. An element $X$ of Freudenthal triple system (FTS) $\mathcal{F}(J)$ 43, 44] over $J$ can be represented formally as a $2 \times 2$ "matrix":

$$
X=\left(\begin{array}{ll}
\alpha & \mathbf{x} \\
\mathbf{y} & \beta
\end{array}\right) \in \mathcal{F}(J)
$$

where $\alpha, \beta \in \mathbb{R}$ and $\mathbf{x}, \mathbf{y} \in J$

Denoting the "bare" four dimensional graviphoton field strength and its magnetic dual as $F_{\mu \nu}^{0}$ and $\widetilde{F}_{0}^{\mu \nu}$, respectively, we have the correspondence

$$
\left(\begin{array}{cc}
F_{\mu \nu}^{0} & F_{\mu \nu}^{I} \\
\widetilde{F}_{I}^{\mu \nu} & \widetilde{F}_{0}^{\mu \nu}
\end{array}\right) \Longleftrightarrow\left(\begin{array}{cc}
e_{0} & e_{I} \\
\tilde{e}^{I} & \tilde{e}^{0}
\end{array}\right) \in \mathcal{F}(J),
$$




\begin{tabular}{|c|c|c|c|}
\hline & $\mathcal{M}_{5}=$ & $\mathcal{M}_{4}=$ & $\mathcal{M}_{3}=$ \\
\hline$J$ & $\operatorname{Str}_{0}(J) / \operatorname{Aut}(J)$ & $\operatorname{Conf}(J) / \widetilde{\operatorname{Str}}(J)$ & QConf $(\mathcal{F}(J)) / \operatorname{Conf}(J) \times \mathrm{SU}(2)$ \\
\hline$J_{3}^{\mathbb{R}}$ & $\mathrm{SL}(3, \mathbb{R}) / \mathrm{SO}(3)$ & $\mathrm{Sp}(6, \mathbb{R}) / \mathrm{U}(3)$ & $\mathrm{F}_{4(4)} / \mathrm{USp}(6) \times \mathrm{SU}(2)$ \\
$J_{3}^{\mathbb{C}}$ & $\mathrm{SL}(3, \mathbb{C}) / \mathrm{SU}(3)$ & $\mathrm{SU}(3,3) / \mathrm{S}(\mathrm{U}(3) \times \mathrm{U}(3))$ & $\mathrm{E}_{6(2)} / \mathrm{SU}(6) \times \mathrm{SU}(2)$ \\
$J_{3}^{\mathbb{H}}$ & $\mathrm{SU}^{*}(6) / \mathrm{USp}(6)$ & $\mathrm{SO}^{*}(12) / \mathrm{U}(6)$ & $\mathrm{E}_{7(-5)} / \mathrm{SO}(12) \times \mathrm{SU}(2)$ \\
$J_{3}^{\mathbb{O}}$ & $\mathrm{E}_{6(-26)} / \mathrm{F}_{4}$ & $\mathrm{E}_{7(-25)} / \mathrm{E}_{6} \times \mathrm{U}(1)$ & $\mathrm{E}_{8(-24)} / \mathrm{E}_{7} \times \mathrm{SU}(2)$ \\
$\mathbb{R} \oplus \Gamma_{(1, n-1)}$ & $\frac{\mathrm{SO}(n-1,1) \times \mathrm{SO}(1,1)}{\mathrm{SO}(n-1)}$ & $\frac{\mathrm{SO}(n, 2) \times \mathrm{SU}(1,1)}{\mathrm{SO}(n) \times \mathrm{SO}(2) \times \mathrm{U}(1)}$ & $\frac{\mathrm{SO}(n+2,4)}{\mathrm{SO}(n+2) \times \mathrm{SO}(4)}$ \\
\hline
\end{tabular}

Table 4: Above we list the scalar manifolds $\mathcal{M}_{d}$ of $N=2$ MESGT's defined by Euclidean Jordan algebras $J$ of degree 3 in $d=3,4,5$ dimensions. $J_{3}^{\mathbb{A}}$ denotes the Jordan algebra of $3 \times 3$ Hermitian matrices over the division algebra $\mathbb{A}=\mathbb{R}, \mathbb{C}, \mathbb{H}, \mathbb{O}$. The last row $\mathbb{R} \oplus \Gamma_{(1, n-1)}$ are the reducible Jordan algebras which are direct sums of Jordan algebras $\Gamma_{(1, n-1)}$ defined by a quadratic form $\mathbb{Q}$ of Minkowskian signature and one dimensional Jordan algebra $\mathbb{R}$. $\widetilde{\operatorname{Str}}(J)$ and $\widetilde{\operatorname{Conf}}(J)$ denote the compact real forms of the structure group $\operatorname{Str}(J)$ and conformal group Conf $(J)$ of a Jordan algebra $J$. QConf $(\mathcal{F}(J))$ denotes the quasiconformal group defined by the FTS $\mathcal{F}(J)$ defined over $J$.

where $e_{I}\left(\tilde{e}^{I}\right)$ are the basis elements of $J$ (its conjugate $\tilde{J}$ ). Consequently, one can associate with a black hole solution with electric and magnetic charges (fluxes) $\left(q_{0}, q_{I}, p^{0}, p^{I}\right)$ of the $4 \mathrm{D}$ MESGT defined by $J$ an element of the FTS $\mathcal{F}(J)$

$$
\left(\begin{array}{cc}
p^{0} e_{0} & p^{I} e_{I} \\
q_{I} \tilde{e}^{I} & q_{0} \tilde{e}^{0}
\end{array}\right) \in \mathcal{F}(J),
$$

U-duality group $G_{4}$ of such a four dimensional MESGT acts as automorphism group of the FTS $\mathcal{F}(J)$, which is endowed with an invariant symmetric quartic form and a skew-symmetric bilinear form. The entropy of an extremal black with charges $\left(p^{0}, p^{I}, q_{0}, q_{I}\right)$ is determined by the quartic invariant $\mathcal{Q}_{4}(q, p)$ of $\mathcal{F}(J)$. With this identification the orbits of extremal black holes of 4D, $N=2$ MESGT's with symmetric scalar manifolds were classified in [14, 45.

Upon further dimensional reduction to three dimensions (C-map) $N=2$ MESGTs lead to $N=4, d=3$ quaternionic Kähler $\sigma$ models coupled to supergravity [6, 46]. In Table 1 we give the symmetry groups of $N=2$ MESGTs defined by Euclidean Jordan algebras in $d=5,4$ and 3 dimensions and their scalar manifolds. We should note that five and three dimensional U-duality symmetry groups $\operatorname{Str}_{0}(J)$ and $Q \operatorname{Con} f(J)$, respectively, act as symmetries of supergravity Lagrangians, while four dimensional U-duality groups $C o n f(J)$ are on-shell symmetries. 


\section{U-duality Orbits of Extremal Black Holes of $4 D, N=2$ MESGTs with Symmetric Scalar Manifolds and of $N=8$ Supergravity and their Spec- trum Generating Quasiconformal Extensions}

The discussion of the orbits extremal black holes of extended supergravity theories with symmetric scalar manifolds were covered in Sergio Ferrara's lectures 47]. Referring to Ferrara's lectures for details including the related recent developments I will briefly summarize the results for $N=2$ MESGTs and $N=8$ supergravity in this section following [45]. As in the five dimensional case, the $4 D$ extremal black hole attractor equations are simply the criticality conditions for the black hole scalar potential which can be written as [48, 29]

$$
V_{B H} \equiv|Z|^{2}+G^{I \bar{J}}\left(D_{I} Z\right)\left(\bar{D} \bar{J}_{\bar{Z}}\right)
$$

where $Z$ is the central charge function. The criticality condition is [30]:

$$
\partial_{I} V_{B H}=0
$$

implies

$$
2 \bar{Z} D_{I} Z+i C_{I J K} G^{J \bar{J}} G^{K \bar{K}} \bar{D} \bar{J} \bar{Z} \bar{K}_{\bar{Z}} \bar{Z}=0
$$

$C_{I J K}$ is the completely symmetric, covariantly holomorphic tensor of special Kähler geometry that satisfies

$$
\bar{D}_{\bar{L}} C_{I J K}=0, \quad D_{[L} C_{I] J K}=0
$$

where square brackets denote antisymmetrization. For symmetric special Kähler manifolds the tensor $C_{I J K}$ is covariantly constant:

$$
D_{I} C_{J K L}=0
$$

which implies the four dimensional counterpart of the adjoint identity [6, 49]

$$
G^{K \bar{K}} G^{M \bar{J}} C_{M(P Q} C_{I J) K} \bar{C}_{\overline{K I J}}=\frac{4}{3} C_{(I J P} G_{Q) \bar{I}}
$$

The $\frac{1}{2}$-BPS attractors are given by the following solution of attractor equations 30]

$$
Z \neq 0, D_{I} Z=0 \quad \forall I=1, \ldots, n_{V} .
$$

The orbits of the 1/2-BPS black hole solutions with positive quartic invariants of $N=2$ MESGTs with symmetric target spaces were given in [14 and are listed in column 1 of Table 5. In [14 a second family of orbits with non-vanishing quartic invariants were also given. They correspond to non-BPS extremal black holes and the respective solutions to the attractor equations were given in [45]. In addition there exist another family of non-BPS extremal black holes with non-vanishing quartic invariant and vanishing central charge [45]. The complete list of orbits of BPS and extremal non-BPS black holes is given in Table 5 . 


\begin{tabular}{|c|c|c|c|}
\hline $\mathrm{J}$ & $\begin{array}{c}\frac{1}{2} \text {-BPS orbits } \\
\mathcal{O}_{\frac{1}{2}-B P S}\end{array}$ & $\begin{array}{l}\text { non-BPS, } Z \neq 0 \text { orbits } \\
\qquad \mathcal{O}_{\text {non }-B P S, Z \neq 0}\end{array}$ & $\begin{array}{c}\text { non-BPS, } Z=0 \text { orbits } \\
\mathcal{O}_{\text {non }-B P S, Z=0}\end{array}$ \\
\hline- & $\frac{S U(1, n+1)}{S U(n+1)}$ & - & $\frac{S U(1, n+1)}{S U(1, n)}$ \\
\hline $\mathbb{R} \oplus \Gamma_{(1, n-1)}$ & $\frac{S U(1,1) \otimes S O(2,2+n)}{S O(2) \otimes S O(2+n)}$ & $\frac{S U(1,1) \otimes S O(2,2+n)}{S O(1,1) \otimes S O(1,1+n)}$ & $\frac{S U(1,1) \otimes S O(2,2+n)}{S O(2) \otimes S O(2, n)}$ \\
\hline$J_{3}^{\mathbb{Q}}$ & $\frac{E_{7(-25)}}{E_{6}}$ & $\frac{E_{7(-25)}}{E_{6(-26)}}$ & $\frac{E_{7(-25)}}{E_{6(-14)}}$ \\
\hline$J_{3}^{\mathbb{H}}$ & $\frac{S O^{*}(12)}{S U(6)}$ & $\frac{S O^{*}(12)}{S U^{*}(6)}$ & $\frac{S O^{*}(12)}{S U(4,2)}$ \\
\hline$J_{3}^{\mathbb{C}}$ & $\frac{S U(3,3)}{S U(3) \otimes S U(3)}$ & $\frac{S U(3,3)}{S L(3, \mathbb{C})}$ & $\frac{S U(3,3)}{S U(2,1) \otimes S U(1,2)}$ \\
\hline$J_{3}^{\mathbb{R}}$ & $\frac{S p(6, \mathbb{R})}{S U(3)}$ & $\frac{S p(6, \mathbb{R})}{S L(3, \mathbb{R})}$ & $\frac{S p(6, \mathbb{R})}{S U(2,1)}$ \\
\hline
\end{tabular}

Table 5: Non-degenerate orbits of $N=2, D=4$ MESGTs with symmetric scalar manifolds. Except for the first row all such theories originate from five dimensions and are defined by Jordan algebras that are indicated in the first column.

The orbits of black hole solutions of $4 D N=8$ supergravity under the action of Uduality group $E_{7(7)}$ were given in [14]. There exist two classes of non-degenerate charge orbits of black hole solutions with non-vanishing quartic invariant $I_{4}$ constructed from the electric and magnetic charges transforming in $\mathbf{5 6}$ of $E_{7(7)}$ [14. Depending on the sign of $I_{4}$, one finds :

$$
\begin{aligned}
& I_{4}>0: \mathcal{O}_{\frac{1}{8}-B P S}=\frac{E_{7(7)}}{E_{6(2)}} \Longleftrightarrow \frac{1}{8} \text {-BPS; } \\
& I_{4}<0: \mathcal{O}_{n o n-B P S}=\frac{E_{7(7)}}{E_{6(6)}} \Longleftrightarrow \text { non-BPS. }
\end{aligned}
$$

This is to be contrasted with the non-degenerate orbits of the exceptional $N=2$ supergravity with U-duality group $E_{7(-25)}$, which has three non-degenerate orbits, one BPS and two non-BPS one of which has vanishing central charge. On the other hand, in $N=8$ super- 
gravity one has $1 / 4$ and $1 / 2$ BPS black holes with vanishing entropy 33. The "light-like" orbits of these BPS black hole solutions with vanishing quartic invariant were given in 14. There are 3 distinct cases depending on the number of vanishing "eigenvalues" that lead to vanishing $\mathcal{Q}_{4}$. The generic light-like orbit for which a single eigenvalue vanishes is

$$
\frac{E_{7(7)}}{F_{4(4)}\left(\mathrm{S} T_{26}\right.}
$$

where $T_{26}$ is a 26 dimensional Abelian subgroup of $E_{7(7)}$ and (S) denotes semi-direct product. The critical light-like orbit has two vanishing eigenvalues and correspond to the 45 dimensional orbit

$$
\frac{E_{7(7)}}{O(6,5)\left(S\left(T_{32} \oplus T_{1}\right)\right.}
$$

The doubly critical light-like orbit with three vanishing eigenvalues is given by the 28 dimensional quotient space

$$
\frac{E_{7(7)}}{\left.E_{6(6)} \mathrm{S}\right) T_{27}}
$$

As discussed above, four dimensional U-duality groups $G_{4}$ were proposed as spectrum generating conformal symmetry groups in five dimensions that leave a cubic light-cone invariant. This raises the question, first investigated in [15], whether the three dimensional U-duality groups $G_{3}$ could act as spectrum generating "conformal" groups of corresponding four dimensional supergravity theories. It is easy to show that there exist three dimensional U-duality groups that do not have any conformal realizations in general. Some other three dimensional U-duality groups do not admit conformal realizations on the $2 n_{V}+2$ dimensional space of the FTS that defines the four dimensional theory. However as was shown in [15] the three dimensional U-duality groups $G_{3}$ all have novel geometric realizations as quasi-conformal groups on the vector spaces of FTS's extended by an extra singlet coordinate that leave invariant a generalized light-cone with respect to a quartic distance function. The quasiconformal actions of three dimensional U-duality groups $G_{3}$ were then proposed as spectrum generating symmetry groups of corresponding four dimensional supergravity theories [15, 35, 50, 34, 51, 52]. We shall denote the quasiconformal groups defined over FTS's $\mathcal{F}$ extended by a singlet coordinate as $Q \operatorname{Conf}(\mathcal{F})$. If the FTS is defined over a Jordan algebra $J$ of degree three we shall denote the corresponding quasiconformal groups either as $Q \operatorname{Conf}(\mathcal{F}(J))$ or simply as $Q \operatorname{Conf}(J)$. The construction given in [15] is covariant with respect to the automorphism group of the FTS, which is isomorphic to the $4 D$ U-duality group of the corresponding supergravity. For $N=2$ MESGTs defined by Jordan algebras of degree three, quasiconformal group actions of their three dimensional U-duality groups $G_{3}$ were given explicitly in [22], in a basis covariant with respect to U-duality groups $G_{6}$ of corresponding six dimensional supergravity theories. 


\section{Quasiconformal Realizations of Lie Groups and Freudenthal Triple Sys- tems}

In this section we shall review the general theory of quasiconformal realizations of noncompact groups over Freudenthal triple systems that was given in [15].

Every simple Lie algebra $\mathfrak{g}$ of dimension greater than three can be given a 5 -graded decomposition $^{5}$, determined by one of its generators $\Delta$, such that grade \pm 2 subspaces are one dimensional:

$$
\mathfrak{g}=\mathfrak{g}^{-2} \oplus \mathfrak{g}^{-1} \oplus \mathfrak{g}^{0} \oplus \mathfrak{g}^{+1} \oplus \mathfrak{g}^{+2}
$$

where

$$
\mathfrak{g}^{0}=\mathfrak{h} \oplus \Delta
$$

and

$$
[\Delta, \mathfrak{t}]=m \mathfrak{t} \quad \forall \mathfrak{t} \in \mathfrak{g}^{m}, m=0, \pm 1, \pm 2
$$

Given such a 5 -graded Lie algebra it can be constructed over a Freudenthal triple system $\mathcal{F}$ which we shall denote as $\mathfrak{g}(\mathcal{F})$ [44, 53]. A Freudenthal triple system (FTS) is defined as a vector space $\mathcal{F}$ equipped with a triple product $(X, Y, Z)$

$$
(X, Y, Z) \in \mathcal{F} \quad \forall X, Y, Z \in \mathcal{F}
$$

that satisfies the identities

$$
\begin{aligned}
(X, Y, Z)= & (Y, X, Z)+2\langle X, Y\rangle Z \\
(X, Y, Z)= & (Z, Y, X)-2\langle X, Z\rangle Y \\
\langle(X, Y, Z), W\rangle= & \langle(X, W, Z), Y\rangle-2\langle X, Z\rangle\langle Y, W\rangle \\
(X, Y,(V, W, Z))= & (V, W,(X, Y, Z)+((X, Y, V), W, Z) \\
& +(V,(Y, X, W), Z) .
\end{aligned}
$$

and admits a skew symmetric bilinear form

$$
\langle X, Y\rangle=-\langle Y, X\rangle \in \mathbb{R}, \quad \forall X, Y \in \mathcal{F}
$$

In the corresponding construction of $\mathfrak{g}(\mathcal{F})$ one labels the generators belonging to subspace $\mathfrak{g}^{+1}$ by the elements of $\mathcal{F}$

$$
U_{A} \in \mathfrak{g}^{+1} \leftrightarrow \quad A \in \mathcal{F}
$$

and through the involution, that reverses the grading, elements of $\mathfrak{g}^{-1}$ can also be labeled by elements of $\mathcal{F}$

$$
\tilde{U}_{A} \in \mathfrak{g}^{-1} \leftrightarrow \quad A \in \mathcal{F}
$$

\footnotetext{
${ }^{5}$ This is to be contrasted with the three grading of generalized conformal groups. No real forms of exceptional Lie algebras $G_{2}, F_{4}$ and $E_{8}$ admit such a three grading.
} 
Elements of $\mathfrak{g}^{ \pm 1}$ generate the full Lie algebra $\mathfrak{g}(\mathcal{F})$ by commutation. The generators belonging to grade zero and grade \pm 2 subspaces are labelled by a pair of elements of $\mathcal{F}$

$$
\begin{aligned}
& {\left[U_{A}, \tilde{U}_{B}\right] \equiv S_{A B} \quad \in \mathfrak{g}^{0}} \\
& {\left[U_{A}, U_{B}\right] \equiv-K_{A B} \in \mathfrak{g}^{2}} \\
& {\left[\tilde{U}_{A}, \tilde{U}_{B}\right] \equiv-\tilde{K}_{A B} \quad \in \mathfrak{g}^{-2}}
\end{aligned}
$$

Commutation relations of the generators of the Lie algebra $\mathfrak{g}$ can all be expressed in terms of the Freudenthal triple product $(A, B, C)$

$$
\begin{aligned}
{\left[S_{A B}, U_{C}\right] } & =-U_{(A, B, C)} \\
{\left[S_{A B}, \tilde{U}_{C}\right] } & =-\tilde{U}_{(B, A, C)} \\
{\left[K_{A B}, \tilde{U}_{C}\right] } & =U_{(A, C, B)}-U_{(B, C, A)} \\
{\left[\tilde{K}_{A B}, U_{C}\right] } & =\tilde{U}_{(B, C, A)}-\tilde{U}_{(A, C, B)} \\
{\left[S_{A B}, S_{C D}\right] } & =-S_{(A, B, C) D}-S_{C(B, A, D)} \\
{\left[S_{A B}, K_{C D}\right] } & =K_{A(C, B, D)}-K_{A(D, B, C)} \\
]\left[S_{A B}, \tilde{K}_{C D}\right] & =\tilde{K}_{(D, A, C) B}-\tilde{K}_{(C, A, D) B} \\
{\left[K_{A B}, \tilde{K}_{C D}\right] } & =S_{(B, C, A) D}-S_{(A, C, B) D}-S_{(B, D, A) C}+S_{(A, D, B) C}
\end{aligned}
$$

Since the grade \pm 2 subspaces are one dimensional their generators can be written as

$$
\begin{aligned}
K_{A B} & :=K_{\langle A, B\rangle}:=\langle A, B\rangle K \\
\tilde{K}_{A B} & :=\tilde{K}_{\langle A, B\rangle}:=\langle A, B\rangle \tilde{K}
\end{aligned}
$$

Now the defining identities of a FTS imply that

$$
S_{A B}-S_{B A}=-2\langle A, B\rangle \Delta
$$

where $\Delta$ is the generator that determines the 5-grading

$$
\begin{aligned}
{\left[\Delta, U_{A}\right] } & =U_{A} \\
{\left[\Delta, \tilde{U}_{A}\right] } & =-\tilde{U}_{A} \\
{[\Delta, K] } & =2 K \\
{[\Delta, \tilde{K}] } & =-2 \tilde{K}
\end{aligned}
$$

and generates a distinguished $\operatorname{sl}(2)$ subalgebra together with $K, \tilde{K}$

$$
[K, \tilde{K}]=-2 \Delta
$$


The 5-grading of $\mathfrak{g}$ can then be recast as

$$
\mathfrak{g}=\tilde{K} \oplus \tilde{U}_{A} \oplus\left[S_{(A B)}+\Delta\right] \oplus U_{A} \oplus K
$$

where

$$
S_{(A B)}:=\frac{1}{2}\left(S_{A B}+S_{B A}\right)
$$

are the generators of the automorphism group $A u t(\mathcal{F})$ of $\mathcal{F}$ that commute with $\Delta$

$$
\left[\Delta, S_{(A B)}\right]=0
$$

The remaining non-zero commutators are

$$
\begin{aligned}
{\left[U_{A}, \tilde{U}_{B}\right] } & =S_{(A B)}-\langle A, B\rangle \Delta \\
{\left[K, \tilde{U}_{A}\right] } & =-2 \tilde{U}_{A} \\
{\left[\tilde{K}, U_{A}\right] } & =2 \tilde{U}_{A} \\
{\left[S_{(A B)}, K\right] } & =0
\end{aligned}
$$

Every FTS $\mathcal{F}$ admits a completely symmetric quadrilinear form which induces a quartic norm $\mathcal{Q}_{4}$. For an element $X \in \mathcal{F}$ the quartic norm is

$$
\mathcal{Q}_{4}(X):=\frac{1}{48}\langle(X, X, X), X\rangle
$$

which is invariant under the automorphism group $A u t(\mathcal{F})$ of $\mathcal{F}$ generated by $S_{(A B)}$.

As was shown in 15] one can realize the 5-graded Lie algebra $\mathfrak{g}$ non-linearly as a quasiconformal Lie algebra over a vector space $\mathcal{T}$ coordinatized by the elements $X$ of the FTS $\mathcal{F}$ plus an extra singlet variable $x$ [15, 22]:

$$
\begin{array}{ccc}
K(X)=0 & U_{A}(X)=A & S_{A B}(X)=(A, B, X) \\
K(x)=2 & U_{A}(x)=\langle A, X\rangle & S_{A B}(x)=2\langle A, B\rangle x \\
\tilde{U}_{A}(X)=\frac{1}{2}(X, A, X)-A x \\
\tilde{U}_{A}(x)=-\frac{1}{6}\langle(X, X, X), A\rangle+\langle X, A\rangle x \\
\tilde{K}(X)=-\frac{1}{6}(X, X, X)+X x \\
\tilde{K}(x)=\frac{1}{6}\langle(X, X, X), X\rangle+2 x^{2}
\end{array}
$$

The quasiconformal action of the Lie algebra $\mathfrak{g}(\mathcal{F})$ on the space $\mathcal{T}$ has a beautiful geometric interpretation. To see this one defines the quartic norm of a vector $\mathcal{X}=(X, x)$ in the space $\mathcal{T}$ as

$$
\mathcal{N}_{4}(\mathcal{X}):=\mathcal{Q}_{4}(X)-x^{2}
$$


where $\mathcal{Q}_{4}(X)$ is the quartic norm of $X \in \mathcal{F}$ and then a "distance" function between any two points $\mathcal{X}=(X, x)$ and $\mathcal{Y}=(Y, y)$ in $\mathcal{T}$ as

$$
d(\mathcal{X}, \mathcal{Y}):=\mathcal{N}_{4}(\delta(\mathcal{X}, \mathcal{Y})
$$

where $\delta(\mathcal{X}, \mathcal{Y})$ is the "symplectic" difference of two vectors $\mathcal{X}$ and $\mathcal{Y}$ :

$$
\delta(\mathcal{X}, \mathcal{Y}):=(X-Y, x-y+\langle X, Y\rangle)=-\delta(\mathcal{Y}, \mathcal{X})
$$

One can then show that the light-like separations with respect to this quartic distance function

$$
d(\mathcal{X}, \mathcal{Y})=0
$$

is left invariant under quasiconformal group action [15]. In other words quasiconformal groups are the invariance groups of "light-cones" defined by a quartic distance function.

\section{0. $3 D$ U-duality Groups as Spectrum Generating Quasiconformal Groups of $4 D$ Supergravity Theories and Quantum Attractor Flows}

As explained above the vector field strengths plus their magnetic duals of a $4 D$ supergravity defined by a Jordan algebra $J$ of degree three are in one-to-one correspondence with the elements of the Freudenthal triple $\mathcal{F}(J)$ defined over $J$. The automorphism group of $\mathcal{F}(J)$ is the U-duality group $G_{4}$ of the supergravity defined by $J$ and is isomorphic to the conformal group $\operatorname{Con} f(J)$ of $J$. Furthermore, U-duality symmetry groups $G_{3}$ of the $3 D$ supergravity theories they reduce to under dimensional reduction are the quasiconformal groups $Q C o n f(J)$ of $\mathcal{F}(J)$. The U-duality groups of $N=2$ MESGTs defined by Jordan algebras of degree three in five, four and three dimensions are also the isometry groups of their scalar manifolds in the respective dimensions. In five dimensions scalar manifolds are

$$
\mathcal{M}_{5}=\frac{\operatorname{Str}_{0}(J)}{\operatorname{Aut}(J)}
$$

where $\operatorname{Str}_{0}(J)$ and $A u t(J)$ are the reduced structure and automorphism groups of $J$, respectively. The scalar manifolds of these theories in four dimensions are

$$
\mathcal{M}_{4}=\frac{\operatorname{Conf}(J)}{\widehat{\operatorname{Str}}_{0}(J) \times U(1)}
$$

where $\operatorname{Con} f(J)$ is the conformal group of the Jordan algebra $J$ and $\widetilde{\operatorname{Str}_{0}}(J)$ is the compact form of the reduced structure group. Upon further dimensional reduction to three dimensions they lead to scalar manifolds of the form

$$
\mathcal{M}_{3}=\frac{Q \operatorname{Conf}(J)}{\widehat{\operatorname{Conf}}(J) \times S U(2)}
$$


where $Q \operatorname{Conf}(J)$ is the quasiconformal group associated with the Jordan algebra $J$ and $\widetilde{\operatorname{Conf}}(J)$ is the compact real form of the conformal group of $J$. The complete list of the symmetric scalar manifolds in five, four and three dimensions are given in Table 1 .

In the original proposal of [15] that the three dimensional U-duality groups act as spectrum generating quasiconformal groups of the corresponding four dimensional supergravity theories the extra singlet coordinate that extends the 56 dimensional charge space $\left(p^{A}, q_{A}\right)$ of black hole solutions of $N=8$ supergravity was interpreted as the entropy $s$ of the black hole. The light cone condition on the 57 dimensional charge-entropy vector $\left(p^{A}, q_{A}, s\right)$ on which $G_{3}$ acts as a quasiconformal group then gives the well-established relation between the entropy $s$ to the quartic invariant $\mathcal{Q}_{4}$ constructed out of the charges

$$
s^{2}=\mathcal{Q}_{4}\left(p^{A}, q_{A}\right)
$$

A concrete and precise implementation of the proposal that three dimensional U-duality groups must act as spectrum generating quasiconformal groups of spherically symmetric stationary BPS black holes of four dimensional supergravity theories, was given in [51, 52, 54] which we will summarize in this section ${ }^{6}$. The basic starting point of these works is the fact that the attractor equations [56, 29] for a spherically symmetric stationary black hole of four dimensional supergravity theories are equivalent to the equations for geodesic motion of a fiducial particle on the moduli space $\mathcal{M}_{3}^{*}$ of the three dimensional supergravity obtained by reduction on a time-like circle. The connection between the stationary black holes of $4 d$ gravity coupled to matter and geodesic motion of a fiducial particle on the pseudo-Riemannian manifold $\mathcal{M}_{3}$ coupled to gravity in three dimensions on a timelike circle was first observed in 57] 7. More specifically, a $4 D$ supergravity theory with symmetric scalar manifold $\mathcal{M}_{4}=G_{4} / K_{4}$ reduces on a space-like circle to a $3 D$ supergravity with scalar manifold

$$
\mathcal{M}_{3}=\frac{G_{3}}{K_{3}}
$$

where $K_{3}$ is the maximal compact subgroup of $G_{3}$. The same theory dimensionally reduced on a time-like circle leads to a theory with scalar manifold of the form 57]

$$
\mathcal{M}^{*}=\frac{G_{3}}{H_{3}}
$$

where $H_{3}$ is a certain noncompact real form of $K_{3}$. Then the stationary, spherically symmetric solutions of the four-dimensional equations of motion are equivalent to geodesic trajectories on the three-dimensional scalar manifold $\mathcal{M}_{3}^{*}=G_{3} / H_{3}$ [57. For $N=2$ MESGTs defined by Euclidean Jordan algebras of degree three the resulting spaces $\mathcal{M}_{3}^{*}$ are para-quaternionic symmetric spaces of the form

$$
\mathcal{M}_{3}^{*}=\frac{Q \operatorname{Conf}(J)}{\operatorname{Conf}(J) \times S U(1,1)}
$$

\footnotetext{
${ }^{6}$ See also [55].

${ }^{7}$ This was used in 58, 59] to construct static and rotating black holes in heterotic string theory.
} 


\begin{tabular}{|c|c|c|c|c|}
\hline$n_{Q}$ & $n_{V}$ & $M_{4}$ & $\mathcal{M}_{3}^{*}$ & $J$ \\
\hline \hline 8 & 1 & $\emptyset$ & $\frac{U(2,1)}{U(1,1) \times U(1)}$ & $\mathbb{R}$ \\
\hline 8 & 2 & $\frac{S L(2, \mathbb{R})}{U(1)}$ & $\frac{G_{2,2}}{S O(2,2)}$ & $\mathbb{R}$ \\
\hline 8 & 7 & $\frac{S p(6, \mathbb{R})}{S U(3) \times U(1)}$ & $\frac{F_{4(4)}}{S p(6, \mathbb{R}) \times S L(2, \mathbb{R})}$ & $J_{3}^{\mathbb{R}}$ \\
\hline 8 & 10 & $\frac{S U(3,3)}{S U(3) \times S U(3) \times U(1)}$ & $\frac{E_{6(2)}}{S U(3,3) \times S L(2, \mathbb{R})}$ & $J_{3}^{\mathbb{C}}$ \\
\hline 8 & 16 & $\frac{S O^{*}(12)}{S U(6) \times U(1)}$ & $\frac{E_{7(-5)}}{S O^{*}(12) \times S L(2, \mathbb{R})}$ & $J_{3}^{\mathbb{H}}$ \\
\hline 8 & 28 & $\frac{E_{7(-25)}}{E_{6} \times U(1)}$ & $\frac{E_{8(-24)}}{E_{7(-25)} \times S L(2, \mathbb{R})}$ & $J_{3}^{\mathbb{O}}$ \\
\hline 8 & $n+2$ & $\frac{S L(2, \mathbb{R})}{U(1)} \times \frac{S O(n, 2)}{S O(n) \times S O(2)}$ & $\frac{S O(n+2,4)}{S O(n, 2) \times S O(2,2)}$ & $\mathbb{R} \oplus \Gamma_{(1, n-1)}$ \\
\hline \hline 16 & $n+2$ & $\frac{S L(2, \mathbb{R})}{U(1)} \times \frac{S O(n-4,6)}{S O(n-4) \times S O(6)}$ & $\frac{S O(n-2,8)}{S O(n-4,2) \times S O(2,6)}$ & $\mathbb{R} \oplus \Gamma_{(5, n-5)}$ \\
\hline \hline 24 & 16 & $\frac{S O^{*}(12)}{S U(6) \times U(1)}$ & $\frac{E_{7(-5)}}{S O^{*}(12) \times S L(2, \mathbb{R}}$ & $J_{3}^{\mathbb{H}}$ \\
\hline 32 & 28 & $\frac{E_{7(7)}}{S U(8)}$ & $\frac{E_{8(8)}}{S O^{*}(16)}$ & $J_{3}^{\mathbb{Q}}$ \\
\hline
\end{tabular}

Table 6: Above we give the number of supercharges $n_{Q}, 4 D$ vector fields $n_{V}$, scalar manifolds of supergravity theories before and after reduction along a timelike Killing vector from $D=4$ to $D=3$, and associated Jordan algebras $J$. Isometry groups of $4 D$ and $3 D$ supergravity theories are given by the conformal, $\operatorname{Conf}(J)$, and quasiconformal groups, $Q \operatorname{Conf}(J)$, of $J$, respectively.

where $\operatorname{Conf}(J)$ is the isometry group of the scalar manifold $\mathcal{M}_{4}$ of the four dimensional theory. In Table 6 we reproduce a table from [51] giving a complete list of supergravity theories whose four dimensional isometry groups are conformal groups $C o n f(J)$ of a Jordan algebra of degree three and the resulting scalar manifolds $\mathcal{M}_{3}^{*}$, which include all $N \geq 4$ supergravity theories as well as $N=2$ MESGTs defined by Euclidean Jordan algebras.

For dimensionally reducing the $D=4$ theory along a timelike direction one makes the standard Kaluza-Klein-type ansatz [57]

$$
d s_{4}^{2}=-e^{2 U}(d t+\omega)^{2}+e^{-2 U} d s_{3}^{2}
$$

which results in $3 D$ Euclidean gravity coupled to scalars, vectors and fermions. Vector fields in three dimensions can be dualized to scalars and the bosonic sector is described simply by the three-dimensional metric $d s_{3}^{2}$ and scalar fields $\phi^{a}$. The three dimensional scalar fields $\phi^{a}$ consist of the scalars coming from the $4 D$ theory, plus electric and magnetic potentials from the reduction of $4 D$ vector fields $A_{t}^{A}$ and their duals, plus the scale factor $U$ and the twist potential dual to the shift $\omega$ defined in equation 10.2. The resulting manifold $\mathcal{M}_{3}^{*}$ of scalar fields can be thought of as analytic continuation of the Riemannian manifold $M_{3}$ obtained from reduction on a space-like circle.

For spherically symmetric configurations, the metric on three-dimensional slices can be written as

$$
d s_{3}^{2}=N^{2}(\rho) d \rho^{2}+r^{2}(\rho)\left[d \theta^{2}+\sin ^{2} \theta d \phi^{2}\right] .
$$


where $\rho$ is the radial coordinate that plays the role of time in radial quantization. The bosonic part of the action then becomes

$$
S=\int d \rho\left[\frac{N}{2}+\frac{1}{2 N}\left(\dot{r}^{2}-r^{2} G_{a b} \dot{\phi}^{a} \dot{\phi}^{b}\right)\right],
$$

where the dot denotes derivative with respect to $\rho$ and $G_{a b}$ is the metric on $\mathcal{M}_{3}^{*}$. Thus four dimensional equations of motion are equivalent to geodesic motion of a fiducial particle on a real cone $\mathbb{R} \times \mathcal{M}_{3}^{*}$ over $\mathcal{M}_{3}^{*}$. The equation of motion for the lapse function $N$, which is an auxiliary field, imposes the Hamiltonian constraint

$$
H=p_{r}^{2}-\frac{1}{r^{2}} G^{a b} p_{a} p_{b}-1 \equiv 0
$$

where $p_{r}$ and $p_{a}$ are the canonical conjugates to $r$ and $\phi^{a}$, respectively. This constraint fixes the mass of the fiducial particle on the cone to be 1. Note that for BPS black holes, one may choose $N=1, \rho=r, p_{r}=1$. With this choice the problem reduces to light-like geodesic motion on $\mathcal{M}_{3}^{*}$, with affine parameter $\tau=1 / r$. The magnetic and electric charges of the black hole are simply Noether charges $P^{A}, Q_{A}$ associated with the generators of $4 D$ gauge transformations in the isometry group $G_{3}$ acting on $\mathcal{M}_{3}^{*}$. These charges generate an Heisenberg subalgebra under Poisson brackets

$$
\left[P^{A}, Q_{B}\right]_{\mathrm{PB}}=2 \delta_{B}^{A} K
$$

where the "central charge" $K$ is the NUT charge of the black hole [39, 51, 52, 60]. ${ }^{8}$ The conserved charge of the isometry that corresponds to rescalings of the time-time component $g_{t t}$ of the metric is the ADM mass $M$ that satisfies

$$
\left[M, P^{A}\right]_{\mathrm{PB}}=P^{A},\left[M, Q_{A}\right]_{\mathrm{PB}}=Q_{A},[M, K]_{\mathrm{PB}}=2 K .
$$

For supergravity theories whose scalar manifolds $\mathcal{M}_{3}^{*}=G_{3} / H_{3}$ are homogeneous or symmetric spaces there exist additional conserved charges associated with the additional isometries. For $N=2$ MESGTs defined by Euclidean Jordan algebras $J$ of degree three the full isometry group of $\mathcal{M}_{3}^{*}$ is the quasiconformal group $Q C o n f(J)$ that has a five grading with respect to the generator $M$

$$
\mathfrak{q} \mathfrak{c o n f}(J)=\tilde{K} \oplus\left(\tilde{P}^{A}, \tilde{Q}_{A}\right) \oplus(\mathfrak{c o n f}(J)+M) \oplus\left(P^{A}, Q_{A}\right) \oplus K
$$

where $\operatorname{con} \mathfrak{f}(J)$ is the Lie algebra of the $4 D$ U-duality group $\operatorname{Con} f(J)$, which commutes with ADM mass generator $M$.

For spherically symmetric stationary solutions, the supersymmetry variation of the fermionic fields $\lambda^{\alpha}$ are of the general form 61]

$$
\delta \lambda^{\alpha}=V_{i}^{\alpha} \epsilon^{i}
$$

\footnotetext{
${ }^{8}$ The solutions with $K \neq 0$ have closed timelike curves when lifted back to four dimensions, as a consequence of the off-diagonal term $\omega=K \cos \theta d \phi$ in the metric (10.2). Therefore real four-dimensional black holes require taking the "central charge" $K \rightarrow 0$ limit.
} 
where $\epsilon^{i}$ is the supersymmetry parameter and $V_{i}^{\alpha}$ is a matrix linear in the velocities $\dot{\phi}^{a} \equiv \frac{\partial \phi}{\partial \tau}$ on $\mathcal{M}_{3}^{*}$. For general $\mathcal{N}=2$ MESGTs reduced to $d=3$, the indices $i=1,2$ and $\alpha=$ $1, \ldots, 2 n_{V}+2$ transform as fundamental representations of the restricted holonomy group $S p(2, \mathbb{R}) \times S p\left(2 n_{V}+2, \mathbb{R}\right)$ of para-quaternionic geometry. For supersymmetric backgrounds this variation vanishes for some non-zero $\epsilon^{i}$. One can show that this is equivalent to the system of equations [39, 51, 52, 60]:

$$
\begin{aligned}
\frac{d z^{I}}{d \tau} & =-e^{U+i \alpha} g^{I \bar{J}} \partial_{\bar{J}}|Z| \\
\frac{d U}{d \tau}+\frac{i}{2} K & =-2 e^{U+i \alpha}|Z|
\end{aligned}
$$

where

$$
Z(P, Q, K)=e^{\mathcal{K} / 2}\left[\left(Q_{A}-2 K \tilde{\zeta}_{A}\right) X^{A}-\left(P^{A}+2 K \zeta^{A}\right) F_{A}\right]
$$

is the central charge function ${ }^{9}$.

For vanishing NUT charge $K$, the above equations take the form of the standard attractor flow equations describing the radial evolution of the scalars towards the black hole horizon [56, 29, 30, 62, 63.

$$
\begin{aligned}
& \frac{d U}{d \tau}=-2 e^{U}|Z| \\
& \frac{d z^{I}}{d \tau}=-e^{U} g^{I \bar{J}} \partial_{\bar{J}}|Z|
\end{aligned}
$$

with the central charge function

$$
Z(P, Q, K=0)=e^{\mathcal{K} / 2}\left[Q_{A} X^{A}-P^{A} F_{A}\right]
$$

The equivalence of attractor flow of $N=2$ supergravity in $d=4$ and supersymmetric geodesic motion on $M_{3}^{*}$ was pointed out in [64].

The scalar fields $\tilde{\zeta}_{A}, \zeta^{A}$ conjugate to the charges $P^{A}$ and $Q_{A}$ evolve according to

$$
\begin{aligned}
\frac{d \zeta^{A}}{d \tau}= & -\frac{1}{2} e^{2 U}\left[(\operatorname{Im} \mathcal{N})^{-1}\right]^{A B} \\
& {\left[Q_{A}-2 K \tilde{\zeta}_{A}-[\operatorname{Re} \mathcal{N}]_{B C}\left(P^{C}+2 K \zeta^{C}\right)\right] } \\
\frac{d \tilde{\zeta}_{A}}{d \tau}= & -\frac{1}{2} e^{2 U}[\operatorname{Im} \mathcal{N}]_{A B}\left(P^{B}+2 K \zeta^{A}\right)-[\operatorname{Re} \mathcal{N}]_{A B} \frac{d \zeta^{J}}{d \tau}
\end{aligned}
$$

where $\mathcal{N}_{A B}$ is the period matrix of special geometry [48].

For $\mathcal{N}=2$ MESGTs defined by Euclidean Jordan algebras $J$ of degree three the holonomy group of $\mathcal{M}_{3}^{*}$ is $\operatorname{Conf}(J) \times S p(2, \mathbb{R}) \subset S p\left(2 n_{V}+4, \mathbb{R}\right)$. The full phase space is $8 n_{V}+8$ dimensional and for BPS black holes supersymmetry leads to $2 n_{V}+1$ first class constraints which reduce the dimension of the phase space to $4 n_{V}+6=\left(8 n_{V}+8\right)-2\left(2 n_{V}+1\right)$. This

\footnotetext{
${ }^{9}$ The phase $\alpha$ is to be chosen such that $d U / d \tau$ is real.
} 
reduced phase of BPS black holes can be identified with the twistor space of $\mathcal{M}_{3}$ of complex dimension $\left(2 n_{V}+3\right)$ 51, 52, 54, 65.

The twistor space of the scalar manifold

$$
\mathcal{M}_{3}=\frac{Q \operatorname{Conf}(J)}{\widehat{\operatorname{Conf}}(J) \times S U(2)}
$$

of dimensionally reduced $N=2$ MESGT defined by a Jordan algebra $J$ is

$$
\mathcal{Z}_{3}=\frac{Q \operatorname{Conf}(J)}{\widehat{\operatorname{Conf}}(J) \times S U(2)} \times \frac{S U(2)}{U(1)}=\frac{Q \operatorname{Conf}(J)}{\widehat{\operatorname{Conf}(J) \times U(1)}}
$$

The quasiconformal group action of a group $G$ extends to its complexification [15]. Consequently, quasiconformal actions of three dimensional U-duality groups $Q \operatorname{Con} f(J)$ on the space with real coordinates $\mathcal{X}=(X, x)$ extend naturally to the complex coordinates $\mathcal{Z}=(Z, z)$ of corresponding twistor spaces $\mathcal{Z}_{3}$ [54. The Kähler potential of the Kähler-Einstein metric of the twistor space is given precisely by the "quartic light-cone" of quasiconformal geometry in these coordinates

$$
\mathcal{K}(\mathcal{Z}, \overline{\mathcal{Z}})=\ln d(\mathcal{Z}, \overline{\mathcal{Z}})=\ln \left[\mathcal{Q}_{4}(\mathbf{Z}-\overline{\mathbf{Z}})+(\mathbf{z}-\overline{\mathbf{z}}+\langle\mathbf{Z}, \overline{\mathbf{Z}}\rangle)^{2}\right]
$$

The Kähler potential is manifestly invariant under the Heisenberg symmetry group corresponding to "symplectic translations" generated by $Q_{A}, P^{A}$ and $K$. Under the global action of "symplectic special conformal generators" $\tilde{Q}_{A}, \tilde{P}^{A}$ and $\tilde{K}$ the quartic light-cone transforms as 15, 54

$$
d(\mathcal{Z}, \overline{\mathcal{Z}}) \Longrightarrow f(\mathbf{Z}, \mathbf{z}) \bar{f}(\overline{\mathbf{Z}}, \overline{\mathbf{z}}) d(\mathcal{Z}, \overline{\mathcal{Z}})
$$

which correspond to Kähler transformations of the Kähler potential 10.17 of the twistor space and hence leaves the Kähler metric invariant. These results were first established for quaternionic symmetric spaces [54 and there exist analogous Kähler potentials for more general quaternionic manifolds invariant only under the Heisenberg symmetries generated by $Q_{A}$, $P^{A}$ and $K$ that are in the C-map [65]. As will be discussed in the next section, the correspondence established between harmonic superspace formulation of $4 D, N=2$ sigma models coupled to $N=2$ supergravity and quasiconformal realizations of their isometry groups [66] implies that Kähler potentials of quartic light-cone type must exist for all quaternionic target manifolds and not only those that are in the C-map.

The quantization of the motion of fiducial particle on $\mathcal{M}_{3}^{*}$ leads to quantum mechanical wave functions that provide the basis of a unitary representation of the isometry group $G_{3}$ of $\mathcal{M}_{3}^{*}$. BPS black holes correspond to a special class of geodesics which lift holomorphically to the twistor space $\mathcal{Z}_{3}$ of $\mathcal{M}_{3}^{*}$. Spherically symmetric stationary BPS black holes of $N=2$ MESGT's are described by holomorphic curves in $\mathcal{Z}_{3}$ [51, 52, 54, 65]. Therefore for theories defined by Jordan algebras $J$ of degree three, the relevant unitary representations of the isometry groups $Q \operatorname{Con} f(J)$ for BPS black holes are those induced by their holomorphic actions on the corresponding twistor spaces $\mathcal{Z}_{3}$, which belong in general to quaternionic 
discrete series representations [54]. For rank two quaternionic groups $S U(2,1)$ and $G_{2(2)}$ unitary representations induced by the geometric quasiconformal actions were studied in great detail in [54].

\section{Harmonic Superspace, Minimal Unitary Representations and Quasicon- formal Group Actions}

In this section we shall review the connection between the harmonic superspace (HSS) formulation of $4 D, N=2$ supersymmetric quaternionic Kähler sigma models that couple to $N=2$ supergravity and the minimal unitary representations of their isometry groups [66. We shall then discuss the relevance of these results to the proposal that quasiconformal extensions of U-duality groups of four dimensional $N=2$ MESGTs must act as spectrum generating symmetry groups [15, 34, 51, 52, 54, which extends the proposal that the conformal extensions of U-duality groups of $N=2, d=5$ MESGTs act as spectrum generating symmetry groups [14, 15, 34].

\section{1 $4 D, N=2 \sigma$-models coupled to Supergravity in Harmonic Superspace}

The target spaces of $N=2$ supersymmetric $\sigma$-models coupled to $N=2$ supergravity in four dimensions are quaternionic Kähler manifolds [61]. They can be formulated in a manifestly supersymmetric form in harmonic superspace [67, 68, 69, 70] which we shall review briefly following [70]. In harmonic superspace approach the metric on a quaternionic target space of $N=2$ sigma model is given by a quaternionic potential $\mathcal{L}^{(+4)}$, which is the analog of Kähler potentials of complex Kähler manifolds.

The $N=2$ harmonic superspace action for the general $4 n$-dimensional quaternionic $\sigma$ model has the simple form $[70]^{10}$

$$
S=\int d \zeta^{(-4)} d u\left\{Q_{\alpha}^{+} D^{++} Q^{+\alpha}-q_{i}^{+} D^{++} q^{+i}+\mathcal{L}^{(+4)}\left(Q^{+}, q^{+}, u^{-}\right)\right\} .
$$

where the integration is over the analytic superspace coordinates $\zeta, u_{i}^{ \pm}$. The hypermultiplet superfields $Q_{\alpha}^{+}(\zeta, u), \alpha=1, \ldots, 2 n$ and the supergravity hypermultiplet compensators

$q_{i}^{+}(\zeta, u),(i=1,2)$ are analytic $N=2$ superfields. The $u_{i}^{ \pm},(i=1,2)$ are the $S^{2}=\frac{S U_{A}(2)}{U_{A}(1)}$ isospinor harmonics that satisfy

$$
u^{+i} u_{i}^{-}=1
$$

and $D^{++}$is a supercovariant derivative with respect to harmonics with the property

$$
D^{++} u_{i}^{-}=u_{i}^{+}
$$

The analytic subspace of the full $N=2$ harmonic superspace involves only half the Grassmann variables with coordinates $\zeta^{M}$ and $u_{i}^{ \pm}$

\footnotetext{
${ }^{10}$ The conventions for indices in this section are independent of the conventions of previous sections and follow closely the conventions used in 66. The number of plus (+) or minus (-) signs in a superscript or subscript denote the $U_{A}(1)$ charges. If the $U_{A}(1)$ charge $n>2$, it is indicated as $(+n)$.
} 


$$
\zeta^{M}:=\left\{x_{A}^{\mu}, \theta^{a+}, \bar{\theta}^{\dot{a}+}\right\}
$$

where

$$
\begin{gathered}
x_{A}^{\mu}:=x^{\mu}-2 i \theta^{(i} \sigma^{\mu} \bar{\theta}^{j)} u_{i}^{+} u_{j}^{-} \\
\theta^{a+}:=\theta^{a i} u_{i}^{+} \\
\bar{\theta}^{\dot{a}+}:=\bar{\theta}^{\dot{a} i} u_{i}^{+} \\
\theta^{(i} \sigma^{\mu} \bar{\theta}^{j)} u_{i}^{+} u_{j}^{-}:=\theta^{(a i}\left(\sigma^{\mu}\right)_{a \dot{a}} \bar{\theta}^{\dot{a} j)} u_{i}^{+} u_{j}^{-} \\
\mu=0,1,2,3 ; a=1,2 ; \dot{a}=1,2
\end{gathered}
$$

Furthermore the analytic subspace does not involve $U(1)$ charge -1 projections of the Grassmann variables and still closes under $N=2$ supersymmetry transformations. It satisfies a "reality condition" with respect to the conjugation $\sim$

$$
\begin{array}{r}
\widetilde{x}^{\mu}=x^{\mu} \\
\widetilde{\theta}^{+}=\bar{\theta}^{+} \\
\widetilde{\bar{\theta}}^{+}=-\theta^{+} \\
\widetilde{u}^{i \pm}=u_{i}^{ \pm} \\
\widetilde{u}_{i}^{ \pm}--u^{\mathrm{i} \pm}
\end{array}
$$

which is a product of complex conjugation and anti-podal map on the sphere $S^{2}$ [71].

The quaternionic potential $\mathcal{L}^{(+4)}$ is a homogeneous function in $Q_{\alpha}^{+}$and $q_{i}^{+}$of degree two and has $U(1)$-charge +4 . It does not depend on $u^{+}$and is, otherwise, an arbitrary "real function" with respect to the involution ? We shall suppress the dependence of all the fields on the harmonic superspace coordinates $\zeta^{M}$ and $u_{i}^{ \pm}$in what follows.

The action 11.1.1 is of the form of Hamiltonian mechanics with the harmonic derivative $D^{++}$playing the role of time derivative $[72,70,71]$ and with the superfields $Q^{+}$and $q^{+}$ corresponding to phase space coordinates under the Poisson brackets

$$
\{f, g\}^{--}=\frac{1}{2} \Omega^{\alpha \beta} \frac{\partial f}{\partial Q^{+\alpha}} \frac{\partial g}{\partial Q^{+\beta}}-\frac{1}{2} \epsilon^{i j} \frac{\partial f}{\partial q^{+i}} \frac{\partial g}{\partial q^{+j}},
$$

$\Omega^{\alpha \beta}$ and $\epsilon^{i j}$ are the invariant antisymmetric tensors of $S p(2 n)$ and $S p(2)$, respectively, which are used to raise and lower indices

$$
\begin{gathered}
Q^{+\alpha}=\Omega^{\alpha \beta} Q_{\beta}^{+} \\
q^{+i}=\epsilon^{i j} q_{j}^{+}
\end{gathered}
$$

and satisfy ${ }^{11}$

\footnotetext{
${ }^{11}$ Note that the conventions of $[7]$ for the symplectic metric , which we follow in this section, differ from those of 73 .
} 


$$
\begin{gathered}
\Omega^{\alpha \beta} \Omega_{\beta \gamma}=\delta_{\gamma}^{\alpha} \\
\epsilon^{i j} \epsilon_{j k}=\delta_{k}^{i}
\end{gathered}
$$

The quaternionic potential $\mathcal{L}^{(+4)}$ plays the role of the Hamiltonian and we shall refer to it as such following [70].

Isometries of the $\sigma$-model 11.1.1 are generated by Killing potentials $K_{A}^{++}\left(Q^{+}, q^{+}, u^{-}\right)$ that obey the conservation law

$$
\partial^{++} K_{A}^{++}+\left\{K_{A}^{++}, \mathcal{L}^{(+4)}\right\}^{--}=0
$$

where $\partial_{++}$is defined as

$$
\partial_{++}=u^{+i} \frac{\partial}{\partial u^{-i}}
$$

They generate the Lie algebra of the isometry group

$$
\left\{K_{A}^{++}, K_{B}^{++}\right\}^{--}=f_{A B}{ }^{C} K_{C}^{++} .
$$

under Poisson brackets 11.1.4.

The "Hamiltonians" $\mathcal{L}^{(+4)}$ of all $N=2 \sigma$-models coupled to $\mathrm{N}=2$ supergravity with irreducible symmetric target manifolds were given in [70]. The quaternionic symmetric spaces, sometimes known as Wolf spaces [74, that arise in $N=2$ supergravity are of the non-compact type. For each simple Lie group there is a unique non-compact quaternionic symmetric space. Below we give a complete list of these spaces:

$$
\begin{gathered}
\frac{S U(n, 2)}{U(n) \times S p(2)} \\
\frac{S O(n, 4)}{S O(n) \times S U(2) \times S p(2)} \\
\frac{U S p(2 n, 2)}{S p(2 n) \times S p(2)} \\
\frac{G_{2(+2)}}{S U(2) \times S p(2)} \\
\frac{F_{4(+4)}}{S p(6) \times S p(2)} \\
\frac{E_{6(+2)}}{S U(6) \times S p(2)} \\
\frac{E_{7(-5)}}{S O(12) \times S p(2)} \\
\frac{E_{8(-24)}}{E_{7} \times S p(2)} .
\end{gathered}
$$

Given a target space $\frac{G}{H \times S p(2)}$ of $N=2 \sigma$ model coordinatized by $Q_{\alpha}^{+}$and $q_{i}^{+}$, every generator $\Gamma_{A}$ of $G$ maps to a function $K_{A}^{++}\left(Q^{+}, q^{+}, u^{-}\right)$such that the action of $K_{A}^{++}$is given 
via the Poisson brackets 11.1.4. Furthermore, it can be shown that the Hamiltonian $\mathcal{L}^{(+4)}$ depends only on $Q_{\alpha}^{+}$and the combination $\left(q^{+} u^{-}\right) \equiv q^{+i} u_{i}^{-}$.70

$$
\mathcal{L}^{(+4)}=\mathcal{L}^{(+4)}\left(Q^{+},\left(q^{+} u^{-}\right)\right)
$$

and can be written as

$$
\mathcal{L}^{(+4)}=\frac{P^{(+4)}\left(Q^{+}\right)}{\left(q^{+} u^{-}\right)^{2}}
$$

The fourth order polynomial $P^{(+4)}$ is given by

$$
P^{(+4)}\left(Q^{+}\right)=\frac{1}{12} S_{\alpha \beta \gamma \delta} Q^{+\alpha} Q^{+\beta} Q^{+\gamma} Q^{+\delta}
$$

where $S_{\alpha \beta \gamma \delta}$ is a completely symmetric invariant tensor of $H$. In terms of matrices $t_{\alpha \beta}^{a}=$ $t_{\beta \alpha}^{a}, a=1,2, \ldots, \operatorname{dim}(H)$ given by the action of the generators $K_{a}^{++}$of $H$ on the coset space generators $K_{i \alpha}^{++}$

$$
\left\{K_{a}^{++}, K_{i \alpha}^{++}\right\}=t_{a \alpha}^{\beta} K_{i \beta}^{++}
$$

the invariant tensor reads as 70

$$
S_{\alpha \beta \gamma \delta}=h_{a b} t_{\alpha \beta}^{a} t_{\gamma \delta}^{b}+\Omega_{\alpha \gamma} \Omega_{\beta \delta}+\Omega_{\alpha \delta} \Omega_{\beta \gamma} .
$$

where $h_{a b}$ is the Killing metric of $H$.

The Killing potentials that generate the isometry group $G$ are given by 70

$$
\begin{gathered}
\mathbf{S p}(\mathbf{2}): \quad K_{i j}^{++}=2\left(q_{i}^{+} q_{j}^{+}-u_{i}^{-} u_{j}^{-} \mathcal{L}^{(+4)}\right), \\
\mathbf{H}: \quad K_{a}^{++}=t_{a \alpha \beta} Q^{+\alpha} Q^{+\beta} \\
\mathbf{G} / \mathbf{H} \times \mathbf{S p}(\mathbf{2}): \quad K_{i \alpha}^{++}=2 q_{i}^{+} Q_{\alpha}^{+}-u_{i}^{-}\left(q^{+} u^{-}\right) \partial_{\alpha}^{-} \mathcal{L}^{(+4)},
\end{gathered}
$$

where

$$
\begin{gathered}
\partial_{\alpha}^{-}:=\frac{\partial}{\partial Q^{+\alpha}} \\
t_{a \alpha \beta}=\Omega_{\beta \gamma} t_{a \alpha}^{\gamma}
\end{gathered}
$$

The Killing potentials $K_{i j}^{++}$that generate $S p(2)$ are conserved for any arbitrary polynomial $P^{(+4)}\left(Q^{+}\right)$. Since $t^{a}$ are also the representation matrices of the generators of $H$ acting on $Q^{+\alpha}$ one finds that the fourth order polynomial $P^{(+4)}$ is proportional to the quadratic "Casimir function" $h^{a b} K_{a}^{++} K_{b}^{++}$of $H$. Furthermore , $P^{(+4)}$ can also be expressed in terms of Killing potentials of the coset space generators, or in terms of the $S p(2)$ Killing potentials as follows [70] :

$$
P^{(+4)}=-\frac{1}{16} \epsilon^{i j} \Omega^{\alpha \beta} K_{i \alpha}^{++} K_{j \beta}^{++}=-\frac{1}{8} K^{++i j} K_{i j}^{++}
$$




\subsection{Minimal Unitary Representations of Non-compact Groups from their Qua- siconformal Realizations}

Before discussing its relationship to the HSS formulation of $N=2$ sigma models given in the previous section, we shall review the unified construction of minimal unitary representations of noncompact groups obtained by quantization of their geometric realizations as quasiconformal groups following [73] which generalizes the results of [75, 76, 22].

Consider the 5 -graded decomposition of the Lie algebra $\mathfrak{g}$ of a noncompact group $G$ of quaternionic type and label the generators such that

$$
\begin{gathered}
\mathfrak{g}=\mathfrak{g}^{-2} \oplus \mathfrak{g}^{-1} \oplus(\mathfrak{h} \oplus \Delta) \oplus \mathfrak{g}^{+1} \oplus \mathfrak{g}^{+2} \\
\mathfrak{g}=E \oplus E^{\alpha} \oplus\left(J^{a}+\Delta\right) \oplus F^{\alpha} \oplus F
\end{gathered}
$$

where $\Delta$ is the generator that determines the 5-grading. Generators $J^{a}$ of $\mathfrak{h}$ satisfy

$$
\left[J^{a}, J^{b}\right]=f_{c}^{a b} J^{c}
$$

where $a, b, \ldots=1, \ldots D=\operatorname{dim}(H)$. We shall denote the symplectic representation by which $\mathfrak{h}$ acts on the subspaces $\mathfrak{g}^{ \pm 1}$ as $\rho$

$$
\left[J^{a}, E^{\alpha}\right]=\left(\lambda^{a}\right)^{\alpha}{ }_{\beta} E^{\beta}, \quad\left[J^{a}, F^{\alpha}\right]=\left(\lambda^{a}\right)^{\alpha}{ }_{\beta} F^{\beta}
$$

where $E^{\alpha}, \alpha, \beta, . .=1, . ., N=\operatorname{dim}(\rho)$ are generators that span the subspace $\mathfrak{g}^{-1}$

$$
\left[E^{\alpha}, E^{\beta}\right]=2 \Omega^{\alpha \beta} E
$$

and $F^{\alpha}$ are generators that span $\mathfrak{g}^{+1}$

$$
\left[F^{\alpha}, F^{\beta}\right]=2 \Omega^{\alpha \beta} F
$$

$\Omega^{\alpha \beta}$ is the symplectic invariant "metric" of the representation $\rho$. The remaining nonvanishing commutation relations of $\mathfrak{g}$ are given by

$$
\begin{array}{rlrl}
F^{\alpha} & =\left[E^{\alpha}, F\right] & {\left[\Delta, E^{\alpha}\right]} & =-E^{\alpha} \\
E^{\alpha} & =\left[E, F^{\alpha}\right] & {\left[\Delta, F^{\alpha}\right]} & =F^{\alpha} \\
{\left[E^{\alpha}, F^{\beta}\right]} & =-\Omega^{\alpha \beta} \Delta+\epsilon \lambda_{a}^{\alpha \beta} J^{a} & {[\Delta, E]} & =-2 E \\
{[\Delta, F]} & =2 F
\end{array}
$$

where $\epsilon$ is a constant parameter whose value depends on the Lie algebra $\mathfrak{g}$. Note that the positive (negative) grade generators form a Heisenberg subalgebra since

$$
\left[E^{\alpha}, E\right]=0
$$

with the grade $+2(-2)$ generator $F(E)$ acting as its central charge. 
In the minimal unitary realization of noncompact groups [73, negative grade generators are expressed as bilinears of symplectic bosonic oscillators $\xi^{\alpha}$ satisfying the canonical commutation relations ${ }^{12}$

$$
\left[\xi^{\alpha}, \xi^{\beta}\right]=\Omega^{\alpha \beta}
$$

and an extra coordinate $y^{13}$.

$$
E=\frac{1}{2} y^{2} \quad E^{\alpha}=y \xi^{\alpha} \quad J^{a}=-\frac{1}{2} \lambda^{a}{ }_{\alpha \beta} \xi^{\alpha} \xi^{\beta}
$$

The quadratic Casimir operator of the Lie algebra $\mathfrak{h}$ is

$$
\mathcal{C}_{2}(\mathfrak{h})=\eta_{a b} J^{a} J^{b}
$$

where $\eta_{a b}$ is the Killing metric of the subgroup $H$, which is isomorphic to the automorphism group of the underlying FTS $\mathcal{F}$. The quadratic Casimir $C_{2}(\mathfrak{h})$ is equal to the quartic invariant of $H$ in the representation $\rho$ modulo an additive constant that depends on the normal ordering chosen, namely

$$
I_{4}\left(\xi^{\alpha}\right)=S_{\alpha \beta \gamma \delta} \xi^{\alpha} \xi^{\beta} \xi^{\gamma} \xi^{\delta}=C_{2}(\mathfrak{h})+\mathfrak{c}
$$

where $\mathfrak{c}$ is a constant and

$$
S_{\alpha \beta \gamma \delta}:=\lambda_{a(\alpha \beta} \lambda_{\gamma \delta)}^{a}
$$

The grade +2 generator $F$ has the general form

$$
F=\frac{1}{2} p^{2}+\frac{\kappa\left(\mathcal{C}_{2}(\mathfrak{h})+\mathfrak{C}\right)}{y^{2}}
$$

where $p$ is the momentum conjugate to the singlet coordinate $y$

$$
[y, p]=i
$$

and $\kappa$ and $\mathfrak{C}$ are some constants depending on the Lie algebra $\mathfrak{g}$. The grade +1 generators are then given by commutators

$$
F^{\alpha}=\left[E^{\alpha}, F\right]=i p \xi^{\alpha}+\kappa y^{-1}\left[\xi^{\alpha}, \mathcal{C}_{2}\right]
$$

If $H$ is simple or Abelian they take the general form 73]

$$
F^{\alpha}=i p \xi^{\alpha}-\kappa y^{-1}\left[2\left(\lambda^{a}\right)^{\alpha}{ }_{\beta} \xi^{\beta} J_{a}+C_{\rho} \xi^{\alpha}\right]
$$

where $C_{\rho}$ is the eigenvalue of the second order Casimir of $H$ in the representation $\rho$. Furthermore, one finds

$$
\left[E^{\alpha}, F^{\beta}\right]=-\Delta \Omega^{\alpha \beta}-6 \kappa\left(\lambda^{a}\right)^{\alpha \beta} J_{a}
$$

\footnotetext{
${ }^{12}$ In this section we follow the conventions of $[73$. The indices $\alpha, \beta, \ldots$ are raised and lowered with the antisymmetric symplectic metric $\Omega^{\alpha \beta}=-\Omega^{\beta \alpha}$ that satisfies $\Omega^{\alpha \beta} \Omega_{\gamma \beta}=\delta_{\beta}^{\alpha}$ and $V^{\alpha}=\Omega^{\alpha \beta} V_{\beta}$, and $V_{\alpha}=V^{\beta} \Omega_{\beta \alpha}$.

${ }^{13}$ In the corresponding geometric quasiconformal realization of the group $G$ over an $(N+1)$ dimensional space this coordinate corresponds to the singlet of $H$
} 
where $\Delta=-\frac{i}{2}(y p+p y)^{14}$.

For simple $H$ the quadratic Casimir operator of the Lie algebra $\mathfrak{g}$ can be calculated simply 73

$$
\mathcal{C}_{2}(\mathfrak{g})=J^{a} J_{a}+\frac{2 C_{\rho}}{N+1}\left(\frac{1}{2} \Delta^{2}+E F+F E\right)-\frac{C_{\rho}}{N+1} \Omega_{\alpha \beta}\left(E^{\alpha} F^{\beta}+F^{\beta} E^{\alpha}\right)
$$

The quadratic Casimir of $\operatorname{sl}(2)$ generated by $E, F$ and $\Delta$ can be expressed in terms of the quadratic Casimir $J^{a} J_{a}$ of $H$ :

$$
\frac{1}{2} \Delta^{2}+E F+F E=\kappa\left(J^{a} J_{a}+\mathfrak{C}\right)-\frac{3}{8}
$$

as well as the contribution of the coset generators $F^{\alpha}$ and $E^{\beta}$ to $\mathcal{C}_{2}(\mathfrak{g})$ to the Casimir of $\mathfrak{g}$

$$
\Omega_{\alpha \beta}\left(E^{\alpha} F^{\beta}+F^{\beta} E^{\alpha}\right)=8 \kappa J^{a} J_{a}+\frac{N}{2}+\kappa C_{\rho} N
$$

Thus the quadratic Casimir of $\mathfrak{g}$ reduces to a c-number 73

$$
\mathcal{C}_{2}(\mathfrak{g})=\mathfrak{C}\left(\frac{8 \kappa C_{\rho}}{N+1}-1\right)-\frac{3}{4} \frac{C_{\rho}}{N+1}-\frac{N}{2} \frac{C_{\rho}}{N+1}-\frac{\kappa C_{\rho}^{2} N}{N+1}
$$

as required by irreducibility of the minimal representation and is a general feature for minimal unitary realizations of all simple groups $G$ obtained from their quasiconformal realizations 75 , 76, 22, 73.

\subsection{Harmonic Superspace Formulation of $N=2$ Sigma Models and Minimal Unitary Representations of Their Isometry Groups}

Let us now show that there is a precise mapping between the Killing potentials of the isometry group $G$ of the sigma model in harmonic superspace and the generators of its minimal unitary realization [66]. This is best achieved by rewriting the Killing potentials in terms of $S U(2)_{A}$ invariant canonical variables, which are defined as follows

$$
\begin{gathered}
\sqrt{2} q^{+i} u_{i}^{-}:=w \\
\sqrt{2} q^{+i} u_{i}^{+}:=p^{++}
\end{gathered}
$$

The poisson brackets of $q^{+i}$

$$
\left\{q^{+i}, q^{+j}\right\}=-\frac{1}{2} \epsilon^{i j}
$$

imply that

$$
\left\{w, p^{++}\right\}=-1
$$

\footnotetext{
${ }^{14}$ In the most general case, where $H$ is not necessarily simple or Abelian, one finds that the commutator of $E^{\alpha}$ and $F^{\beta}$ has the same form as above.
} 
Under the conjugation $\sim$ we have

$$
\begin{aligned}
& \underset{q^{+i}}{\widetilde{\widetilde{d}}}=-q^{+i} \\
& \underset{\widetilde{u_{i}^{ \pm}}}{\widetilde{ }}=-u_{i}^{ \pm}
\end{aligned}
$$

which imply

$$
\begin{gathered}
\widetilde{\widetilde{w}}=w \\
\widetilde{\widetilde{p^{++}}}=p^{++}
\end{gathered}
$$

The Hamiltonian can then be written as

$$
\mathcal{L}^{(+4)}=\frac{2 P^{(+4)}\left(Q^{+}\right)}{w^{2}}
$$

The $S U_{A}(2)$ invariant Killing potentials that generate the isometry group $G$ are then

$$
\begin{array}{lll}
\mathbf{S p}(\mathbf{2}): & M^{(+4)}:=M^{++++}=K_{i j}^{++} u^{+i} u^{+j}=\left(p^{++}\right)^{2}-\frac{2 P^{(+4)}\left(Q^{+}\right)}{w^{2}} \\
& M^{++} & =K_{i j}^{++}\left(u^{+i} u^{-j}+u^{+j} u^{-i}\right)=w p^{++}+p^{++} w \\
& M^{0} & =K_{i j}^{++} u^{-i} u^{-j}=w^{2} \\
& & \\
& K_{a}^{++} & =t_{a \alpha \beta} Q^{+\alpha} Q^{+\beta} \\
\mathbf{G} / \mathbf{H} \times \mathbf{S p}(\mathbf{2}): & T^{(+3)} & :=T_{\alpha}^{+++}=K_{i \alpha}^{++} u^{+i}=-\sqrt{2}\left\{p^{++} Q_{\alpha}^{+}-\frac{\partial_{\alpha}^{-} P^{(+4)}\left(Q^{+}\right)}{w}\right\} \\
& T_{\alpha}^{+} & =K_{i \alpha}^{++} u^{-i}=-\sqrt{2} w Q_{\alpha}^{+}
\end{array}
$$

Comparing the above Killing potentials of the isometry group $G$ with the generators of the minimal unitary realization of $G$ given above we have one-to-one correspondence between the elements of harmonic superspace (HSS) and those of minimal unitary realizations (MINREP) given in Table 0

The Poisson brackets (PB) $\{$,$\} in HSS formulation go over to i$ times the commutator [,] in the minimal unitary realization and the harmonic superfields $w, p^{++}$corresponding to supergravity hypermultiplet compensators, that are canonically conjugate under PB map to the canonically conjugate coordinate and momentum operators $y, p$. Similarly, the harmonic superfields $Q^{+\alpha}$ that form $N / 2$ conjugate pairs under Poisson brackets map into the symplectic bosonic oscillators $\xi^{\alpha}$ on the MINREP side. One finds a normal ordering ambiguity in the quantum versions of the quartic invariants. The classical expression relating the quartic invariant polynomial $P^{(+4)}$ to the quadratic Casimir function in HSS formulation differs from the expression relating the quartic quantum invariant $I_{4}$ to the quadratic Casimir of $H$ by an additive c-number depending on the ordering chosen. The consistent choices for the quadratic Casimirs for all noncompact groups of quaternionic type were given in 76, 22, 73].

Furthermore, the mapping between HSS formulation of $N=2$ sigma models and minimal unitary realizations of their isometry groups $G$ extends also to the equations relating the 


\begin{tabular}{||c|c||}
\hline \hline$\sigma$-model with Isometry Group $G$ in HSS & Minimal Unitary Representation of $G$ \\
\hline$w$ & $y$ \\
\hline$p^{++}$ & $p$ \\
\hline$\{\}$, & $i[]$, \\
\hline$Q^{+\alpha}$ & $\xi^{\alpha}$ \\
\hline$P^{(+4)}\left(Q^{+}\right)$ & $I_{4}(\xi)$ \\
\hline$K^{a++}=t_{\alpha \beta}^{a} Q^{+\alpha} Q^{+\beta}$ & $J^{a}=\lambda_{\alpha \beta}^{a} \xi^{\alpha} \xi^{\beta}$ \\
\hline$T_{\alpha}^{+++}=K_{i \alpha}^{++} u^{+i}$ & $F^{\alpha}$ \\
\hline$T_{\alpha}^{+}=K_{i \alpha}^{++} u^{-i}$ & $E^{\alpha}$ \\
\hline$M^{++++}$ & $F$ \\
\hline$M^{0}$ & $E$ \\
\hline$M^{++}$ & $\Delta$ \\
\hline \hline
\end{tabular}

Table 7: Above we give the correspondence between the quantities of the harmonic space formulation of $N=2$ sigma models coupled to supergravity and the operators that enter in the minimal unitary realizations of their isometry groups.

quadratic Casimir of the subgroup $H$ to the quadratic Casimir of $S p(2)$ subgroup and to the contribution of the coset generators $G / H \times S p(2)$ to the quadratic Casimir of $G$ modulo some additive constants due to normal ordering.

On the MINREP side we are working with a realization in terms of quantum mechanical coordinates and momenta, while in HSS side the corresponding quantities are classical harmonic analytic superfields. The above correspondence can be extended to the full quantum correspondence on both sides by reducing the $4 D N=2 \sigma$ model to one dimension and quantizing it to get a supersymmetric quantum mechanics ( with 8 supercharges). The bosonic spectrum of the corresponding quantum mechanics must furnish a minimal unitary representation of the isometry group, which extends to a fully supersymmetric spectrum.

\subsection{Implications}

The mapping between the formulation of $N=2, d=4$ quaternionic Kähler $\sigma$ models in 
HSS and the minimal unitary realizations of their isometry groups reviewed above is quite remarkable. It implies that the fundamental spectra of the quantum $N=2$, quaternionic Kähler $\sigma$ models in $d=4$ and their lower dimensional counterparts must fit into the minimal unitary representations of their isometry groups. The fundamental spectra consist of states created by the action of harmonic analytic superfields at a given point in analytic superspace with coordinates $\zeta^{M}$ on the vacuum of the theory. The above analysis shows that the states created by purely bosonic components of analytic superfields will fit into the minimal unitary representation of the corresponding isometry group. Since the analytic superfields are unconstrained, the bosonic spectrum must extend to full $N=2$ supersymmetric spectrum ( 8 supercharges) by the action of the fermionic components of the superfields.

The minimal unitary representations for general noncompact groups are the analogs of the singleton representations of symplectic groups $S p(2 n, \mathbb{R})$ 73]. The singleton realizations of $S p(2 n, \mathbb{R})$ correspond to free field realizations, i.e. their generators can be written as bilinears of bosonic oscillators ${ }^{15}$. As a consequence the tensoring procedure becomes simple and straightforward for the symplectic groups [77]. However, in general, the minimal unitary realization of a noncompact group is "interacting" and the corresponding generators are nonlinear in terms of the coordinates and momenta, which makes the tensoring problem highly nontrivial. For the quantum $N=2$ quaternionic Kähler $\sigma$ models one then has to tensor the fundamental supersymmetric spectra with each other repeatedly to obtain the full 'perturbative" spectra. The full "nonperturbative" spectra in quantum HSS will, in general, contain states that do not form full $N=2$ supermultiplets, such as $1 / 2$ BPS black holes etc...

We should also stress that the fundamental spectrum is generated by the action of analytic harmonic superfields involving an infinite number of auxiliary fields. Once the auxiliary fields are eliminated the dynamical components of the superfields become complicated nonlinear functions of the physical bosonic and fermionic fields. Therefore the "fundamental spectrum" in HSS is in general not the simple Fock space of free bosons and fermions.

The $N=2, d=4$ MESGT's under dimensional reduction lead to $N=4, d=3$ supersymmetric $\sigma$ models with quaternionic Kähler manifolds $\mathcal{M}_{3}$ (C-map). After T-dualizing the three dimensional theory one can lift it back to four dimension, thereby obtaining an $N=2$ sigma model coupled to supergravity that is in the mirror image of the original $N=2$ MESGT. In previous sections we discussed how quantizing spherically symmetric stationary BPS black hole solutions of $4 D, N=2$ MESGTs defined by Jordan algebras naturally lead to quaternionic discrete series representations of their three dimensional U-duality groups $Q \operatorname{Con} f(J)$. The results summarized in this section suggest even a stronger result, namely, quantized solutions (spectra) of a $4 D, N=2$ MESGT, that allow dimensional reduction to three dimensions, must fit into the minimal unitary representation of its three dimensional U-duality group and those representations obtained by tensoring of minimal representations.

\footnotetext{
${ }^{15}$ For symplectic groups the quartic invariant vanishes and hence the minimal unitary realization becomes a free field construction.
} 


\begin{tabular}{|c|c|c|c|}
\hline$d$ & $J_{3}^{\otimes}$ MESGT & $N=8$ Supergravity & $J_{3}^{\mathbb{H}}$ MESGT \\
\hline 5 & $E_{6(-26)}$ & $E_{6(6)}$ & $S U^{*}(6)$ \\
\hline 4 & $E_{7(-25)}$ & $E_{7(7)}$ & $S O^{*}(12)$ \\
\hline 3 & $E_{8(-24)}$ & $E_{8(8)}$ & $E_{7(-5)}$ \\
\hline
\end{tabular}

Table 8: Above we give the U-duality groups of the exceptional $N=2$ MESGT defined by the exceptional Jordan algebra and of the maximal $N=8$ supergravity in five, four and three spacetime dimensions. In the last column we list the U-duality group of the $N=2$ MESGT defined by $J_{3}^{\mathbb{H}}$ that is a common sector of these two theories.

\section{M/Superstring Theoretic Origins of $N=2$ MESGTs with Symmetric Scalar Manifolds}

Both the maximal supergravity and the exceptional $N=2$ MESGT defined by the exceptional Jordan algebra $J_{3}^{\mathbb{D}}$ have exceptional groups of the $E$ series as their U-duality symmetry groups in five, four and three dimensions. The numbers of vector fields of these two theories are the same in five and four dimensions. However, the real forms of their U-duality groups are different. In the Table 8 we list the U-duality groups of the $N=2$ exceptional MESGT and those of maximal $N=8$ supergravity. Just like the $N=8$ supergravity the fields of the exceptional $N=2$ MESGT could not be identified with quarks and leptons so as to obtain a unified theory of all interactions without invoking a composite scenario [5, 78]. However, it was observed that unlike the maximal $N=8$ supergravity theory one might be able to couple matter multiplets to the exceptional supergravity theory which could be identified with quarks and leptons [5, 78]. These two theories have a common sector which is the $N=2$ MESGT defined by the quaternionic Jordan algebra $J_{3}^{\mathbb{H}}[5]$. The existence of the exceptional MESGT begs the question whether there exists a larger theory that can be "truncated" to both the exceptional MESGT and the maximal supergravity theory [78]. After the discovery of Green-Schwarz anomaly cancellation and the development of Calabi-Yau technology this question evolved into the question whether one could obtain the exceptional $4 D$ and $5 D$ MESGTs as low energy effective theories of type II superstring theory or M-theory on some exceptional Calabi-Yau manifold [8]. In fact more generally one would like to know if $N=2$ MESGTs with symmetric target spaces can be obtained from M/superstring theory on some Calabi-Yau manifold with or without hypermultiplet couplings. The compactifications of M/Superstring theory over generic Calabi-Yau manifolds do not , in general, lead to symmetric or homogeneous scalar manifolds in the corresponding five or four dimensional theories [79, 80]. In the mathematics literature this was posed as the question whether Hermitian symmetric spaces could arise as moduli spaces of deformations of Hodge structures of Calabi-Yau manifolds [81]. In particular, the difficulty of obtaining the exceptional moduli space $\frac{E_{7(-25)}}{E_{6} \times U(1)}$ was highlighted by Gross [81].

Using methods developed earlier in [82] Sen and Vafa constructed dual pairs of compactifications of type II superstring with $N=2,4$ and $N=6$ supersymmetries in $d=4$ by orbifolding $T^{4} \times S^{1} \times S^{1}[83$. Remarkably, the low energy effective theory of one of the com- 
pactifications they study is precisely the magical $N=2$ MESGT defined by the quaternionic Jordan algebra $J_{3}^{\mathbb{H}}$ without any hypermultiplets [84, 85]. This follows from the fact that the resulting $N=2$ theory has the same bosonic field content as the $N=6$ supergravity which they also obtain via orbifolding. As was shown in [5], the bosonic sector of $N=6$ supergravity and the $N=2$ MESGT defined by $J_{3}^{\mathbb{H}}$ are identical and their scalar manifold in $d=4$ is $S O^{*}(12) / U(6)^{16}$. This theory is self-dual with the dilaton belonging to a vector multiplet. In addition to the magical $N=2$ MESGT defined by $J_{3}^{\mathbb{H}}$, Sen and Vafa gave several other examples of compactifications with $N=2$ supersymmetry and symmetric target manifolds in $d=4$. One is the STU model coupled to 4 hypermultiplets with scalar manifold

$$
\mathcal{M}_{V} \times \mathcal{M}_{H}=\left[\frac{S U(1,1)}{U(1)}\right]^{3} \times \frac{S O(4,4)}{S O(4) \times S O(4)}
$$

which is also self-dual. Another theory they construct leads to $N=2$ supergravity belonging to the generic Jordan family defined by the Jordan algebra $J=\mathbb{R} \oplus \Gamma_{(1,6)}$ with the target space

$$
\mathcal{M}_{V}=\frac{S O(6,2) \times S U(1,1)}{S O(6) \times U(1) \times U(1)}
$$

which is not self-dual.

A well-known theory with a symmetric target space that descends from type II string theory is the FSHV model [86]. It is obtained by compactification on a self-mirror Calabi-Yau manifold with Hodge numbers $h^{(1,1)}=h^{(2,1)}=11$ and has the $4 D$ scalar manifold

$$
\mathcal{M}_{V} \times \mathcal{M}_{H}=\frac{S O(10,2) \times S U(1,1)}{S O(10) \times U(1) \times U(1)} \times \frac{S O(12,4)}{S O(12) \times S O(4)}
$$

corresponding to the MESGT defined by the Jordan algebra $\left(\mathbb{R} \oplus \Gamma_{(1,9)}\right)$ coupled to 12 hypermultiplets.

It was known for sometime [87 that there exists a six dimensional $(1,0)$ supergravity theory, which is free from gravitational anomalies, with 16 vector multiplets, 9 tensor multiplets and 28 hypermultiplets, that parametrize the exceptional quaternionic symmetric space $E_{8(-24)} / E_{7} \times S U(2)^{17}$. This theory reduces to the exceptional supergravity theory defined by $J_{3}^{\mathbb{Q}}$ coupled to the hypermultiplets in lower dimensions. It has the $4 D$ scalar manifold

$$
\mathcal{M}_{V} \times \mathcal{M}_{H}=\frac{E_{7(-25)}}{E_{6} \times U(1)} \times \frac{E_{8(-24)}}{E_{7} \times S U(2)}
$$

and in three dimensions the moduli space becomes

$$
\frac{E_{8(-24)}}{E_{7} \times S U(2)} \times \frac{E_{8(-24)}}{E_{7} \times S U(2)}
$$

\footnotetext{
${ }^{16}$ The authors of 83] appear to be unaware of this fact. It is easy to show that the resulting $N=2$ supergravity with 15 vector multiplets can not belong to the generic Jordan family with the scalar manifold $\frac{S O(14,2) \times S U(1,1)}{S O(14) \times U(1) \times U(1)}$.

${ }^{17}$ This theory remains anomaly free if one replaces the hypermultiplet sector with any 112 ( real) dimensional quaternionic sigma model.
} 
The moduli space of the FSHV model is a subspace of this doubly exceptional moduli space. The existence of an anomaly free theory in $d=6$ that reduces to this doubly exceptional theory whose moduli space includes that of the FSHV model suggests strongly that there must exist an exceptional Calabi-Yau manifold such that $\mathrm{M} /$ superstring theory compactified over it yields this doubly exceptional theory as was argued in 84, 85].

Some important developments about the stringy origins of magical supergravity theories that took place after SAM 2007 is summarized in the next section.

\section{Recent Developments and Some Open Problems}

Since they were delivered, a great deal of progress has been made on the main topics covered in these lecture, which I will try to summarize briefly in this section.

We discussed at some length the proposals that four and three dimensional U-duality groups act as spectrum generating conformal and quasiconformal groups of five and four dimensional supergravity theories with symmetric scalar manifolds respectively. Applying these proposals in succession implies that three dimensional U-duality groups should act as spectrum generating symmetry groups of the five dimensional theories from which they descend. Several results that have been obtained recently lend further support to these proposals. The authors of [88, 89] used solution generating techniques to relate the known black hole solutions of simple five dimensional ungauged supergravity and those of the $5 D$ STU model to each other and generate new solutions by the action of corresponding $3 D$ Uduality groups $G_{2(2)}$ and $S O(4,4)$, respectively. For simple ungauged $5 D$ supergravity similar results were also obtained in 90] and for gauged $5 D$ supergravity in 91.

Attractor flows for non-BPS extremal black holes that are described by certain pseudoRiemannian symmetric sigma models coupled to $3 D$ supergravity was carried out in 92$]^{18}$. Extremal BPS and non-BPS black holes of supergravity theories with symmetric target spaces were also studied in [94, 95]. For a more complete up-to-date list of references on extremal BPS and non-BPS attractors and their orbit structures I refer to 47].

As we discussed above the quantization of the attractor flows of stationary spherically symmetric $4 D N=2$ BPS black holes leads to wave-functions that form the basis of quaternionic discrete series representations of the corresponding $3 D$ U-duality groups $Q C o n f(J)$. Unitary representations of two quaternionic groups of rank two, namely $S U(2,1)$ and $G_{2(2)}$, induced by their geometric quasiconformal actions were studied in [54]. The starting point of the constructions of unitary representations given in [54] are the spherical vectors with respect to the maximal compact subgroups $S U(2) \times U(1)$ and $S U(2) \times S U(2)$, respectively, under their quasiconformal actions. In a recent paper with Pavlyk [96] we gave a unified realization of three dimensional U-duality groups of all $N=2$ MESGTs defined by Euclidean Jordan algebras of degree three as spectrum generating quasiconformal groups covariant with respect to their $5 D$ U-duality groups. The spherical vectors of quasiconformal realizations of

\footnotetext{
${ }^{18}$ Preliminary results of this work was reported by Li [93] in this school after these lectures were delivered.
} 
all these groups twisted by a unitary character with respect to their maximal compact subgroups as well as their quadratic Casimir operators and their values were also given in [96]. In a subsequent paper [97 we extended these results to a unified realization of split exceptional groups $F_{4(4)}, E_{6(6)}, E_{7(7)}, E_{8(8)}$ and of $S O(n+3, m+3)$ as quasiconformal groups that is covariant with respect to their subgroups $S L(3, R), S L(3, R) \times S L(3, R), S L(6, R), E_{6(6)}$ and $S O(n, m) \times S O(1,1)$, respectively, and determined their spherical vectors. We also gave their quadratic Casimir operators and determined their values in terms of $\nu$ and the dimension $n_{V}$ of the underlying Jordan algebras. For $\nu=-\left(n_{V}+2\right)+i \rho$ the quasiconformal action induces unitary representations on the space of square integrable functions in $\left(2 n_{V}+3\right)$ variables, that belong to the principle series. For special discrete values of $\nu$ the quasiconformal action leads to unitary representations belonging to the discrete series and their continuations. For the quaternionic real forms of $3 D$ U-duality groups these discrete series representations belong to the quaternionic discrete series.

As I discussed in section 11, the minimal unitary representations of non-compact groups are the analogs of the singleton representations of the symplectic groups $S p(2 n, \mathbb{R})$. Now $S p(4, \mathbb{R})$ is the covering group $4 D$ anti-de Sitter or $3 D$ conformal group $S O(3,2)$. For higher AdS or conformal groups the analogous representations were referred to as doubletons [98, 99]. Singleton or doubleton representations of AdS or conformal groups $S O(d, 2)$ are singular positive energy ( lowest weight) unitary representations, which are in the continuation of holomorphic discrete representations. Even though they do not belong to the holomorphioc discrete series, by tensoring singletons or doubletons one can obtain the entire holomorphic discrete series representations of AdS or conformal groups. This fact lies at the heart of AdS/CFT dualities [100]. In fact, the entire K-K spectrum of IIB supergravity over $A d S_{5} \times S^{5}$ was obtained by tensoring the CPT invariant doubleton supermultiplet repeatedly with itself in [99. Again it was pointed out in [99] that the doubleton supermultiplet decouples from the $\mathrm{K}-\mathrm{K}$ spectrum as gauge modes and its field theory is the conformally invariant $N=4$ super Yang-Mills theory that lives on the boundary of $A d S_{5}$ which is the $4 D$ Minkowski space. Similar results were obtained for 11-dimensional supergravity on $A d S_{4} \times S^{7}$ [101 and on $A d S_{7} \times S^{4}$ 98.

Similarly, the minimal unitary representations of noncompact groups of quaternionic type do not belong to the quaternionic discrete series, but are in their singular continuations [102]. I argued in section 11 that the "fundamental spectrum" of $N=2$ sigma models coupled to supergravity with symmetric target manifolds $G / K \times S U(2)$ must belong to the minimal unitary representation of $G$ obtained by quantization of the geometric quasiconformal realization of $G$. On the other hand quantization of the geodesic motion describing the dynamics of spherically symmetric BPS black holes lead to wave functions belonging to the quaternionic discrete series representations of $G$ [54]. This implies that by tensoring minimal unitary representations one should be able to obtain the quaternionic discrete series representations. Decomposition of tensor products of minimal unitary representations into its irreducible pieces is much harder for general noncompact groups since their minimal representations are , in general, not of the lowest weight type and their realizations are non-linear ( interacting)! This problem is 
currently under investigation 103 .

Several novel results were obtained regarding the stringy origins of magical supergravity theories since SAM 2007. In [104] the hyper-free $N=2$ string models based on asymmetric orbifolds with $N=(4,1)$ world-sheet superconformal symmetry using $2 D$ fermionic construction were given. Among these models two of them correspond to two of the magical supergravity theories, namely the $J_{3}^{\mathbb{C}}$ MESGT with the moduli space

$$
\mathcal{M}_{4}=\frac{S U(3,3)}{S U(3) \times S U(3) \times U(1)}
$$

and the $J_{3}^{\mathbb{H}}$ theory with the moduli space

$$
\mathcal{M}_{4}=\frac{S O^{*}(12)}{U(6)}
$$

In [105], Bianchi and Ferrara reconsidered the string derivation of FSHV model over the Enriques Calabi-Yau and argued that the exceptional supergravity theory defined by octonionic $J_{3}^{\mathbb{Q}}$ admits a string interpretation closely related to the Enriques model. They show that the uplift of the exceptional MESGT to $d=6$ has 16 Abelian vectors which is related to the rank of Type I and heterotic strings.

In mathematics literature, Todorov gave a construction of Calabi-Yau n-folds whose moduli spaces are locally symmetric spaces [106]. This family of CY manifolds of complex dimension $n$ are double covers of the projective $n$ dimensional spaces ramified over $2 n+2$ hyperplanes. They have the Hodge numbers

$$
h^{(n-p, p)}=\left(\begin{array}{l}
n \\
p
\end{array}\right)^{2}
$$

and have the second Betti numbers

$$
b_{2}=\left(\begin{array}{c}
(2 n+2) \\
2
\end{array}\right)+1
$$

Type IIB superstring theory compactified over such a CY threefold constructed by Todorov leads to the magical $N=2$ MESGT defined by the complex Jordan algebra $J_{3}^{\mathbb{C}}$ with vector moduli space

$$
\mathcal{M}_{4}=\frac{S U(3,3)}{S U(3) \times S U(3) \times U(1)}
$$

coupled to $\left(h^{(1,1)}+1\right)=30$ hypermultiplets. Todorov promises a sequel to his paper in which he would show that there are no instanton corrections to the moduli space of this Calabi-Yau manifold.

The fact that some $N=2$ MESGTs with symmetric target spaces, in particular the magical supergravity theories, can be obtained from M/Superstring theory is of utmost importance. It implies that the corresponding supergravity theories have quantum completions and 
discrete arithmetic subgroups of their U-duality groups will survive at the non-perturbative regime of M/Superstring theory [3]. The relevant unitary representations of the spectrum generating symmetry groups will then be some " automorphic representations". If and how some of the results obtained for continuous U-duality groups extend to their discrete subgroups is a wide open problem.

Another open problem is the quantization of $4 D, N=2$ sigma models that couple to supergravity in harmonic superspace and their lower dimensional descendents.

Acknowledgements: I would like to thank Stefano Bellucci for his kind invitation to deliver these lectures at SAM 2007 and the participants for numerous stimulating discussions. I wish to thank Stefano Bellucci, Sergio Ferrara, Kilian Koepsell, Alessio Marrani, Andy Neitzke, Hermann Nicolai, Oleksandr Pavlyk, Boris Pioline and Andrew Waldron for enjoyable collaborations and stimulating discussions on various topics covered in these lectures. Thanks are also due to the organizers of the "Fundamental Aspects of Superstring Theory 2009" Workshop at KITP, UC Santa Barbara and of the New Perspectives in String Theory 2009 Workshop at GGI, Florence where part of these lectures were written up. This work was supported in part by the National Science Foundation under grant number PHY0555605. Any opinions, findings and conclusions or recommendations expressed in this material are those of the authors and do not necessarily reflect the views of the National Science Foundation.

\section{References}

[1] W. Nahm, "Supersymmetries and their representations," Nucl. Phys. B135 (1978) 149.

[2] E. Cremmer, B. Julia, and J. Scherk, "Supergravity theory in 11 dimensions," Phys. Lett. B76 (1978) 409-412.

[3] C. M. Hull and P. K. Townsend, "Unity of superstring dualities," Nucl. Phys. B438 (1995) 109-137, hep-th/9410167.

[4] N. Marcus and J. H. Schwarz, "Three-Dimensional Supergravity Theories," Nucl. Phys. B228 (1983) 145.

[5] M. Günaydin, G. Sierra, and P. K. Townsend, "Exceptional supergravity theories and the magic square," Phys. Lett. B133 (1983) 72.

[6] M. Günaydin, G. Sierra, and P. K. Townsend, "The geometry of $\mathcal{N}=2$ Maxwell-Einstein supergravity and Jordan algebras," Nucl. Phys. B242 (1984) 244.

[7] M. Günaydin, G. Sierra, and P. K. Townsend, "Gauging the $d=5$ Maxwell-Einstein supergravity theories: more on Jordan algebras," Nucl. Phys. B253 (1985) 573.

[8] M. Günaydin, G. Sierra, and P. K. Townsend, "More on d=5 Maxwell-Einstein supergravity: symmetric spaces and kinks," Class. Quant. Grav. 3 (1986) 763.

[9] B. de Wit, F. Vanderseypen, and A. Van Proeyen, "Symmetry structure of special geometries," Nucl. Phys. B400 (1993) 463-524, hep-th/9210068. 
[10] D. Kazhdan and A. Polishchuk, "Minimal representations: spherical vectors and automorphic functionals," in Algebraic groups and arithmetic, pp. 127-198. Tata Inst. Fund. Res., Mumbai, 2004.

[11] K. McCrimmon, A taste of Jordan algebras. Universitext. Springer-Verlag, New York, 2004.

[12] N. Jacobson, Structure and representations of Jordan algebras. American Mathematical Society Colloquium Publications, Vol. XXXIX. American Mathematical Society, Providence, R.I., 1968 .

[13] M. Günaydin and F. Gürsey, "Quark structure and octonions," J. Math. Phys. 14 (1973) $1651-1667$.

[14] S. Ferrara and M. Günaydin, "Orbits of exceptional groups, duality and BPS states in string theory," Int. J. Mod. Phys. A13 (1998) 2075-2088, hep-th/9708025.

[15] M. Günaydin, K. Koepsell, and H. Nicolai, "Conformal and quasiconformal realizations of exceptional Lie groups," Commun. Math. Phys. 221 (2001) 57-76, hep-th/0008063.

[16] M. Günaydin, "Exceptional realizations of Lorentz group: Supersymmetries and leptons," Nuovo Cim. A29 (1975) 467.

[17] V. G. Kac, "Lie Superalgebras," Adv. Math. 26 (1977) 8-96.

[18] M. Günaydin, "Quadratic Jordan formulation of quantum mechanics and construction of Lie (super)algebras from Jordan (super)algebras," Ann. Israel Phys. Soc. (1980), no. 3, 279.

Presented at 8th Int. Colloq. on Group Theoretical Methods in Physics, Kiriat Anavim, Israel, Mar 25-29, 1979.

[19] M. Günaydin, "The exceptional superspace and the quadratic Jordan formulation of quantum mechanics,". In "Elementary particles and the universe: Essays in honor of Murray Gell-Mann", Pasadena 1989, pp. 99-119., ed. by J. Schwarz, Cambridge University Press.

[20] M. Günaydin, "Generalized conformal and superconformal group actions and Jordan algebras," Mod. Phys. Lett. A8 (1993) 1407-1416, hep-th/9301050.

[21] G. Sierra, "An application to the theories of Jordan algebras and Freudenthal triple systems to particles and strings," Class. Quant. Grav. 4 (1987) 227.

[22] M. Günaydin and O. Pavlyk, "Generalized spacetimes defined by cubic forms and the minimal unitary realizations of their quasiconformal groups," JHEP 08 (2005) 101, hep-th/0506010.

[23] M. Günaydin, "AdS/CFT dualities and the unitary representations of non-compact groups and supergroups: Wigner versus Dirac," hep-th/0005168.

[24] G. Mack and M. de Riese, "Simple space-time symmetries: Generalizing conformal field theory," hep-th/0410277.

[25] I. L. Kantor, "Certain generalizations of Jordan algebras," Trudy Sem. Vektor. Tenzor. Anal. 16 (1972) 407-499.

[26] J. Tits, "Une classe d'algèbres de Lie en relation avec les algèbres de Jordan," Nederl. Akad. Wetensch. Proc. Ser. A 65 = Indag. Math. 24 (1962) 530-535.

[27] M. Koecher, "Imbedding of Jordan algebras into Lie algebras. II," Amer. J. Math. 90 (1968) 476-510. 
[28] S. Ferrara and M. Gunaydin, "Orbits and attractors for $N=2$ Maxwell-Einstein supergravity theories in five dimensions," Nucl. Phys. B759 (2006) 1-19, hep-th/0606108.

[29] S. Ferrara and R. Kallosh, "Universality of supersymmetric attractors," Phys. Rev. D54 (1996) 1525-1534, hep-th/9603090.

[30] S. Ferrara, G. W. Gibbons, and R. Kallosh, "Black holes and critical points in moduli space," Nucl. Phys. B500 (1997) 75-93, hep-th/9702103.

[31] S. Ferrara and R. Kallosh, "On N = 8 attractors," Phys. Rev. D73 (2006) 125005, hep-th/0603247.

[32] A. H. Chamseddine, S. Ferrara, G. W. Gibbons, and R. Kallosh, "Enhancement of supersymmetry near 5d black hole horizon," Phys. Rev. D55 (1997) 3647-3653, hep-th/9610155.

[33] S. Ferrara and J. M. Maldacena, "Branes, central charges and $U$-duality invariant BPS conditions," Class. Quant. Grav. 15 (1998) 749-758, hep-th/9706097.

[34] M. Günaydin, "Unitary realizations of U-duality groups as conformal and quasiconformal groups and extremal black holes of supergravity theories," AIP Conf. Proc. 767 (2005) 268-287, hep-th/0502235.

[35] M. Günaydin, "Realizations of exceptional U-duality groups as conformal and quasiconformal groups and their minimal unitary representations," Comment. Phys. Math. Soc. Sci. Fenn. 166 (2004) 111-125, hep-th/0409263.

[36] D. Gaiotto, A. Strominger, and X. Yin, "5d black rings and 4d black holes," JHEP 02 (2006) 023, hep-th/0504126.

[37] D. Gaiotto, A. Strominger, and X. Yin, "New connections between 4d and 5d black holes," JHEP 02 (2006) 024, hep-th/0503217.

[38] H. Elvang, R. Emparan, D. Mateos, and H. S. Reall, "Supersymmetric 4D rotating black holes from $5 \mathrm{D}$ black rings," hep-th/0504125.

[39] B. Pioline, "BPS black hole degeneracies and minimal automorphic representations," JHEP 0508 (2005) 071, hep-th/0506228.

[40] B. de Wit and A. Van Proeyen, "Special geometry, cubic polynomials and homogeneous quaternionic spaces," Commun. Math. Phys. 149 (1992) 307-334, hep-th/9112027.

[41] M. Günaydin, S. McReynolds, and M. Zagermann, "The R-map and the coupling of N = 2 tensor multiplets in 5 and 4 dimensions," JHEP 01 (2006) 168, hep-th/0511025.

[42] M. Koecher, The Minnesota notes on Jordan algebras and their applications, vol. 1710 of Lecture Notes in Mathematics. Springer-Verlag, Berlin, 1999. Edited, annotated and with a preface by Aloys Krieg and Sebastian Walcher.

[43] H. Freudenthal, "Lie groups in the foundations of geometry," Advances in Math. 1 (1964), no. fasc. $2,145-190$.

[44] H. Freudenthal, "Beziehungen der $E_{7}$ und $E_{8}$ zur Oktavenebene. I," Nederl. Akad. Wetensch. Proc. Ser. A. $\mathbf{5 7}$ = Indagationes Math. 16 (1954) 218-230. 
[45] S. Bellucci, S. Ferrara, M. Gunaydin, and A. Marrani, "Charge orbits of symmetric special geometries and attractors," Int. J. Mod. Phys. A21 (2006) 5043-5098, hep-th/0606209.

[46] S. Ferrara and S. Sabharwal, "Quaternionic manifolds for type II superstring vacua of Calabi-Yau spaces," Nucl. Phys. B332 (1990) 317.

[47] S. Bellucci, S. Ferrara, M. Gunaydin, and A. Marrani, "SAM Lectures on Extremal Black Holes in d=4 Extended Supergravity," 0905.3739.

[48] A. Ceresole, R. D'Auria, and S. Ferrara, "The symplectic structure of $\mathcal{N}=2$ supergravity and its central extension," Nucl. Phys. Proc. Suppl. 46 (1996) 67-74, hep-th/9509160.

[49] E. Cremmer and A. Van Proeyen, "Classification of Kahler manifolds in $\mathcal{N}=2$ vector multiplet supergravity couplings," Class. Quant. Grav. 2 (1985) 445.

[50] M. Günaydin, "Realizations of exceptional U-duality groups as conformal and quasi-conformal groups and their minimal unitary representations,". Prepared for 3rd International Symposium on Quantum Theory and Symmetries (QTS3), Cincinnati, Ohio, 10-14 Sep 2003.

[51] M. Günaydin, A. Neitzke, B. Pioline, and A. Waldron, "BPS black holes, quantum attractor flows and automorphic forms," Phys. Rev. D73 (2006) 084019, hep-th/0512296.

[52] M. Günaydin, A. Neitzke, B. Pioline, and A. Waldron, "Quantum Attractor Flows," JHEP 09 (2007) 056, 0707.0267.

[53] I. Kantor and I. Skopets, "Some results on Freudenthal triple systems," Sel. Math. Sov. 2 (1982) 293.

[54] M. Günaydin, A. Neitzke, O. Pavlyk, and B. Pioline, "Quasi-conformal actions, quaternionic discrete series and twistors: $S U(2,1)$ and $G_{2(2)}, "$ Commun. Math. Phys. 283 (2008) 169-226, 0707.1669 .

[55] B. Pioline, "Lectures on black holes, topological strings and quantum attractors (2.0)," Lect. Notes Phys. 755 (2008) 1-91.

[56] S. Ferrara, R. Kallosh, and A. Strominger, "N $\mathcal{N}=2$ extremal black holes," Phys. Rev. D52 (1995) 5412-5416, hep-th/9508072.

[57] P. Breitenlohner, G. W. Gibbons, and D. Maison, "Four-dimensional black holes from Kaluza-Klein theories," Commun. Math. Phys. 120 (1988) 295.

[58] M. Cvetic and D. Youm, "All the static spherically symmetric black holes of heterotic string on a six torus," Nucl. Phys. B472 (1996) 249-267, hep-th/9512127.

[59] M. Cvetic and D. Youm, "Dyonic bps saturated black holes of heterotic string on a six torus," Phys. Rev. D53 (1996) 584-588, hep-th/9507090.

[60] M. Günaydin, A. Neitzke, and B. Pioline, "Topological wave functions and heat equations," JHEP 12 (2006) 070, hep-th/0607200.

[61] J. Bagger and E. Witten, "Matter couplings in $\mathcal{N}=2$ supergravity," Nucl. Phys. B222 (1983) 1.

[62] G. W. Moore, "Arithmetic and attractors," hep-th/9807087.

[63] F. Denef, "Supergravity flows and D-brane stability," JHEP 08 (2000) 050, hep-th/0005049. 
[64] M. Gutperle and M. Spalinski, "Supergravity instantons for $\mathcal{N}=2$ hypermultiplets," Nucl. Phys. B598 (2001) 509-529, hep-th/0010192.

[65] A. Neitzke, B. Pioline, and S. Vandoren, "Twistors and Black Holes," JHEP 04 (2007) 038, hep-th/0701214.

[66] M. Günaydin, "Harmonic Superspace, Minimal Unitary Representations and Quasiconformal Groups," JHEP 05 (2007) 049, hep-th/0702046.

[67] A. Galperin, E. Ivanov, S. Kalitsyn, V. Ogievetsky, and E. Sokatchev, "Unconstrained N=2 Matter, Yang-Mills and Supergravity Theories in Harmonic Superspace," Class. Quant. Grav. 1 (1984) 469-498.

[68] J. A. Bagger, A. S. Galperin, E. A. Ivanov, and V. I. Ogievetsky, "GAUGING N=2 SIGMA MODELS IN HARMONIC SUPERSPACE," Nucl. Phys. B303 (1988) 522.

[69] A. Galperin, E. Ivanov, and O. Ogievetsky, "Harmonic space and quaternionic manifolds," Ann. Phys. 230 (1994) 201-249, hep-th/9212155.

[70] A. Galperin and O. Ogievetsky, "Harmonic potentials for quaternionic symmetric sigma models," Phys. Lett. B301 (1993) 67-71, hep-th/9210153.

[71] A. S. Galperin, E. A. Ivanov, V. I. Ogievetsky, and E. S. Sokatchev, "Harmonic superspace,". Cambridge, UK: Univ. Pr. (2001) 306 p.

[72] A. Galperin and V. Ogievetsky, " $\mathrm{N}=2 \mathrm{D}=4$ supersymmetric sigma models and Hamiltonian mechanics," Class. Quant. Grav. 8 (1991) 1757-1764.

[73] M. Günaydin and O. Pavlyk, "A unified approach to the minimal unitary realizations of noncompact groups and supergroups," JHEP 09 (2006) 050, hep-th/0604077.

[74] J. A. Wolf, "Complex homogeneous contact manifolds and quaternionic symmetric spaces," $J$. Math. Mech. 14 (1965) 1033-1047.

[75] M. Günaydin, K. Koepsell, and H. Nicolai, "The minimal unitary representation of $E_{8(8)}$," Adv. Theor. Math. Phys. 5 (2002) 923-946, hep-th/0109005.

[76] M. Günaydin and O. Pavlyk, "Minimal unitary realizations of exceptional U-duality groups and their subgroups as quasiconformal groups," JHEP 01 (2005) 019, hep-th/0409272.

[77] M. Günaydin and C. Saclioglu, "Oscillator-like unitary representations of noncompact groups with a Jordan structure and the noncompact groups of supergravity," Commun. Math. Phys. 87 (1982) 159.

[78] M. Gunaydin, "EXCEPTIONAL SUPERGRAVITY THEORIES, JORDAN ALGEBRAS AND THE MAGIC SQUARE,". Presented at 13th Int. Colloq. on Group Theoretical Methods in Physics, College Park, MD, May 21-25, 1984.

[79] P. Candelas, G. T. Horowitz, A. Strominger, and E. Witten, "Vacuum Configurations for Superstrings," Nucl. Phys. B258 (1985) 46-74.

[80] A. C. Cadavid, A. Ceresole, R. D'Auria, and S. Ferrara, "Eleven-dimensional supergravity compactified on Calabi-Yau threefolds," Phys. Lett. B357 (1995) 76-80, hep-th/9506144.

[81] B. H. Gross, "A remark on tube domains," Math. Res. Lett. 1 (1994), no. 1, 1-9. 
[82] C. Vafa and E. Witten, "Dual string pairs with $\mathrm{N}=1$ and $\mathrm{N}=2$ supersymmetry in four dimensions," Nucl. Phys. Proc. Suppl. 46 (1996) 225-247, hep-th/9507050.

[83] A. Sen and C. Vafa, "Dual pairs of type II string compactification," Nucl. Phys. B455 (1995) 165-187, hep-th/9508064.

[84] M. Günaydin. talk at IAS, Princeton, Sept 2006.

[85] M. Günaydin. talk titled " From d=6, N=1 to $\mathrm{d}=4, \mathrm{~N}=2$, No-scale models and Jordan Algebras" at the Conference 30 Years of Supergravity in Paris, October, 2006 : http://cft.igc.psu.edu/research/index.shtml\#gunaydin.

[86] S. Ferrara, J. A. Harvey, A. Strominger, and C. Vafa, "Second quantized mirror symmetry," Phys. Lett. B361 (1995) 59-65, hep-th/9505162.

[87] M. Günaydin and E. Sezgin. , unpublished.

[88] A. Bouchareb et al., " $G_{2}$ generating technique for minimal $\mathrm{D}=5$ supergravity and black rings," Phys. Rev. D76 (2007) 104032, 0708.2361.

[89] D. V. Gal'tsov and N. G. Scherbluk, "Generating technique for $U(1)^{3}, 5 D$ supergravity," 0805.3924 .

[90] G. Compere, S. de Buyl, E. Jamsin, and A. Virmani, "G2 Dualities in D=5 Supergravity and Black Strings," Class. Quant. Grav. 26 (2009) 125016, 0903.1645.

[91] M. Berkooz and B. Pioline, "5D Black Holes and Non-linear Sigma Models," JHEP 05 (2008) $045,0802.1659$.

[92] D. Gaiotto, W. W. Li, and M. Padi, "Non-Supersymmetric Attractor Flow in Symmetric Spaces," JHEP 12 (2007) 093, 0710.1638.

[93] W. Li, "Non-Supersymmetric Attractors in Symmetric Coset Spaces," 0801.2536.

[94] E. Bergshoeff, W. Chemissany, A. Ploegh, M. Trigiante, and T. Van Riet, "Generating Geodesic Flows and Supergravity Solutions," Nucl. Phys. B812 (2009) 343-401, 0806.2310.

[95] G. Bossard, H. Nicolai, and K. S. Stelle, "Universal BPS structure of stationary supergravity solutions," 0902.4438.

[96] M. Gunaydin and O. Pavlyk, "Spectrum Generating Conformal and Quasiconformal U-Duality Groups, Supergravity and Spherical Vectors," 0901.1646.

[97] M. Gunaydin and O. Pavlyk, "Quasiconformal Realizations of $E_{6(6)}, E_{7(7)}, E_{8(8)}$ and $S O(n+3, m+3), N=4$ and $N>4$ Supergravity and Spherical Vectors," 0904.0784.

[98] M. Gunaydin, P. van Nieuwenhuizen, and N. P. Warner, "GENERAL CONSTRUCTION OF THE UNITARY REPRESENTATIONS OF ANTI-DE SITTER SUPERALGEBRAS AND THE SPECTRUM OF THE $S^{4}$ COMPACTIFICATION OF ELEVEN-DIMENSIONAL SUPERGRAVITY," Nucl. Phys. B255 (1985) 63.

[99] M. Gunaydin and N. Marcus, "The Spectrum of the $S^{5}$ Compactification of the Chiral $N=2, D=10$ Supergravity and the Unitary Supermultiplets of $U(2,2 / 4), "$ Class. Quant. Grav. 2 (1985) L11.

[100] J. M. Maldacena, "The large N limit of superconformal field theories and supergravity," Adv. Theor. Math. Phys. 2 (1998) 231-252, hep-th/9711200. 
[101] M. Gunaydin and N. P. Warner, "UNITARY SUPERMULTIPLETS OF $O S p(8 / 4, R)$ AND THE SPECTRUM OF THE $S^{7}$ COMPACTIFICATION OF ELEVEN-DIMENSIONAL SUPERGRAVITY," Nucl. Phys. B272 (1986) 99.

[102] B. H. Gross and N. R. Wallach, "On quaternionic discrete series representations, and their continuations," J. Reine Angew. Math. 481 (1996) 73-123.

[103] M. Günaydin., in preparation.

[104] Y. Dolivet, B. Julia, and C. Kounnas, "Magic N=2 supergravities from hyper-free superstrings," JHEP 02 (2008) 097, 0712.2867.

[105] M. Bianchi and S. Ferrara, "Enriques and Octonionic Magic Supergravity Models," JHEP 02 (2008) 054, 0712.2976.

[106] A. N. Todorov, "CY manifolds with locally symmetric moduli spaces," arXiv:0806.4010. 